\title{
NOUVELLES ÉPAVES DE PROVENCE
}

\author{
par M. Fernand BenoIT
}

L'inventaire des épaves reconnues sur la côte de Provence s'est enrichi de plusieurs gisements : ils ont d'autant plus d'intérêt qu'ils renferment un trésor de timbres amphoriques, dont l'équivalent n'avait encore été rencontré qu'au Grand Congloué .

Le rythme de ces découvertes s'accroît malheureusement à une cadence plus rapide que le développement des moyens techniques et financiers dont il faudrait disposer pour assurer la conservation et la protection de ces documents, et la sauvegarde même des épaves, trop souvent livrécs au pillage et dispersées dès l'annonce de leur découverte.

1. Épave du Titan à l'Ile du Levant. Cette épave découverte par le $D^{r}$ Piroux en 1948, à la pointe N.-E. de l'île par 27 mètres de fond, au pied de l'écueil des Esquillades², a été au cours de l'été 1957 l'objet d'une fouille méthodique par le Cap. de Frégate $\mathrm{Ph}$. Tailliez. Il avait à sa disposition les puissants moyens de l'Arsenal de Toulon, en particulier un chaland de la Marine Nationale (fig. 1), équipé d'un compresseur pour le dévasage avec tuyaux de deux diamètres différents et d'une cabine-laboratoire. Le personnel ćtait fourni par l'École de déminage.

Le gisement, dont la couche supérieure avait été écrémée au cours des annécs précédentes ${ }^{3}$, était en bon état, l'épave reposant cntre des rochers sur un lit de sable, dans une position à peu près horizontale. Les amphores, inclinées lors du naufrage, étaient juxtaposées les unes à côté des autres, sans doute sur deux lits seulement ; 700 amphores, dont la plus grande partie intactes, ont été remontées et sont entreposées à la Tour Royale de Toulon, dépendant des Musécs de la Marine.

L'épave peut être datée du milieu du ${ }^{\text {er }}$ siècle avant notre ère par le synchronisme de la céramique campanienne, de lampes de la fin de la République et de deux as semi-onciaux très corrodés; l'un, lisible en creux inversé sur la

(1) Voir nos articles précédents : Amphores et céramiques de l'épave de Marseille, Gallia, XII1954, p. 35-54 (Grand Congloué); Épaves de la côte de Provence : typologie des amphores, XIV-1956, pp. 23-34 (inventaire des épaves).

(2) Gallia, XIV-1956, p. 29, no 10 et fig. 1, nos 11-14

(3) Photo du gisement avant le pillage en 1955 : ibid., p. 31, fig. 3. 
concrétion (fig. 3), porte au revers la proue de navire à droite (diam. max. $0,029)$; pesant 11 gr. 8 , il esl donc postérieur à la réforme monétaire de 89 qui avait fixé lc poids de l'as à 13 gr. $50^{4}$; l'autre, illisible et amoindri, pèse 9 gr. 7 .

Amphores. - Du même type ${ }^{5}$, mais de cinq formats différents, d'autant plus hautes qu'elles sont plus eflilées ( 1 mètre à $1 \mathrm{~m}$. 10), elles sont caractérisées par leur



Fig. 1. - Chaland-laboratoire du $\mathrm{C}^{\imath}$ Tailliez sur le chantier de l'épave du Titan. Pholo $C^{\mathrm{t}}$ Tailliez.

embouchure évasée; elles appartiennent au type 12 de Dressel, précisé par le 48 d'E. Pélichet ${ }^{6}$, daté de l'époque d'Auguste.

Certaines avaient contenu de la saumure de poisson, qui parait avoir été répartie selon la nature de la conserve : têtes et queues, quartiers de chair avec vertèbres de thonidés et pélamides, gastéropodes ptéropodes ont été trouvés dans des amphores

(4) E. Babelon, Monnaies Rép. romaine, I, p. 75. La pièce est trop usée pour une détermination précise : elle est antérieure au G. B. d'Auguste, frappé à I yon portant au revers le vaisseau (H. CoHEN, Méd. impériales, no 737); il semble que l'avers soit à l'effigie de Janus lauré : le diamètre et le style font penser à l'as de la gens Clovia (Babelon, I, p. 361).

(5) La prospection de 1955 avait remonté une amphore à huile du type ovoïde à anses bifides, conservée au C A S M de Cannes : Gallia, XIV-1956, p. 30, n 10 et fig. $1, \mathrm{n}^{\circ} 14$; dans le lot remonté en 1957 (ibid., fig. $1, \mathrm{n}^{\mathrm{ns}} 1 \mathrm{l}-13$ ), une amphore incomplete du col, du type Dressel $1 \mathrm{~B}$, à epaulement horizontal (type infra, nos 2 et 7) qui confirme la datation proposée.

(6) Amphores romaines trouvées à Nyon, Rev. suisse d'arl et d'arch., 1946, p. 193, fig. 6. 

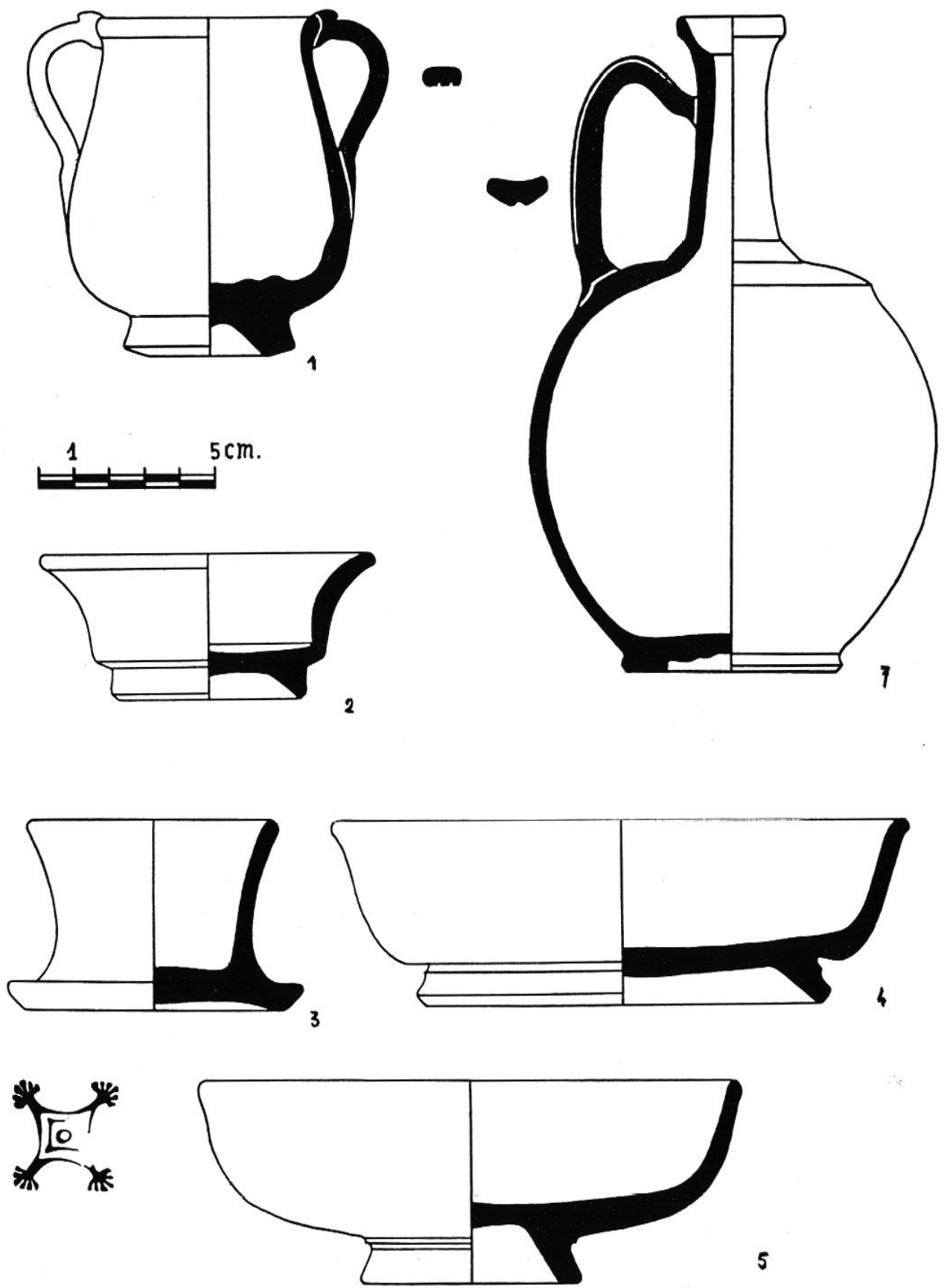

5

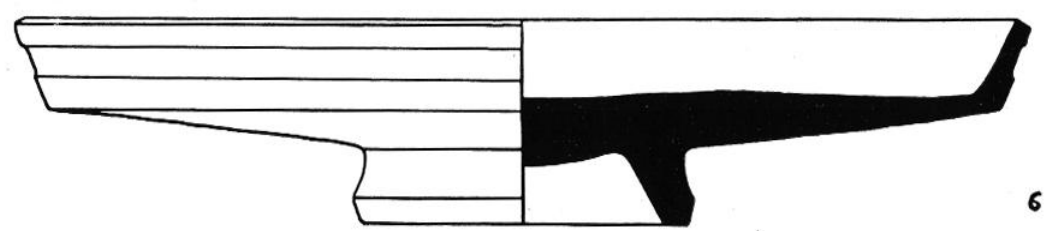

Fig. 2. - Poterie campanienne (type B) et olpé : 1. Petite urne à deux anses -2 et 3. Pyxis - 4. Tasse plate à paroi incurvée -5 . Tasse plate décorée de palmettes liées en losange -6 . Patère à rebord vertical, décorée de cercles incisés. - 7. Olpé en terre commune. $1: 2$. Relevés Ch. Lagrand. 
différentes. La fermeture était faite avec un bouchon de liège et un opercule de terre cuite en forme de couvercle avec tenon de préhension; d'autres amphores contenaient des amandes. Des ossements de mouton (épiphyses) ont été recueillis par la dévaseuse : salaison de conserve ou viande fraiche?

La forme générale de l'amphore est comparable à celle de l'«amphore d'Augst ", à embouchure tronconique d'un large diamètre trouvée en abondance dans les camps du limes (Dressel 6/14; Pélichet $46^{7}$ ); si l'on observe que les marques peintes sur ces récipients indiquent qu'ils contenaient de la saumure (garum scombri) ${ }^{8}$, on peut en déduire que ce type d'amphore se différencie par son embouchure évasée "en entonnoir" de



Fig. 3. - Monnaie de bronze. Proue de navire du revers, d'après le moule en creux de la concrétion. 1 : 1. Phato Y. Rigoir. l'amphore vinaire, caractérisée par le type cylindrique à col droit Dressel 1. Aucune de ces amphores ne porte de marque.

Céramique. - La poterie comprend des olpés (fig. 2, 7) el des cruches en terre commune et de la céramique campanienne, du type $\mathrm{B}$ à pâte gris-rose et vernis mat (fig. 2), caractéristique du milieu du I ${ }^{\text {er }}$ siècle avant J.-C.9 :

pyxis ou coupe à embouchure évasée et pied oblique (forme 2 de Lamboglia); pyxis à paroi évasée de forme concave (forme 3 de Lamboglia) ; tasse plate, sans anse, à paroi incurvée, dérivée du skyphos (forme 1 de Lamboglia), dont un modèle porte des palmettes liées, dégénérées ; patère à rebord vertical, et fond plat (forme 5 de Lamboglia), prototype de la patère arétine (Dragendorff 17), décorée de cercles concentriques incisés ; petite urne à deux anses, en forme de skyphos (forme 10 de Lamboglia);

deux lampes à ailettes à réservoir circulaire tronconique à bec court relevé en proue, avec anse annulaire (type 3 de Dressel) ${ }^{10}$; elles appartiennent à deux modèles différents : l'une à petites ailettes triangulaires, à bec coupé horizontalement (fig. 4); l'autre à ailettes cornues, à bec légèrement incurvé (fig. 5) qui annonce le " bec à enclume " du début du I ${ }^{\text {er }}$ siècle ; toutes deux, en argile rose couverte d'un brun rouge, sont dépourvues de figures ; le disque de la première est orné de rais partant du trou d'alimentation, la seconde a un décor "granulé " et comporte un petit trou à la base du disque pour régler la mèche; des fragments de tuiles plates, à rebord en quart de rond, d'un profil légèrement plus aplati que celui des tuiles du Grand Congloué, caractéristiques de la fin de l'époque républicaine; la présence de quelques-unes de ces tuiles dans les épaves de Marseille et $d^{\prime}$ Albenga ${ }^{11}$ montre qu'elles ne faisaient pas partie du fret, mais avaient servi à la couverture de la cabine de poupe du "château arrière ».

(7) Ibid., p. 192, fig. 3.

(8) A. Grenier, Manuel, II, p. 615, fig. 210, 1 et p. 636, fig. 225, 5 et 6 ; cf. infra à Fos.

(9) Cf. sur la typologie de la campanienne: N. Lambogla, Per una classificazione preliminare della ceramica campana, dans Atti del $I^{\circ}$ Congresso internaz. di Studi liguri, 1950, p. 143 à 149.

(10) Cf. P. de Palol Salfillas, Lucernas romanas... en el museo arqueologico de Gerona, dans Memorias de los Museos arqueolog. provinciales, IX-X, 1948-49, p. 234, fig. 101, no8 9, 10 et p. 241 (de la république à Auguste); S. J. de LakT, Les fouilles d'Alba Fucens (1951-53), Bruxelles, 1955, p. 124, fig. 32 , nos 7-8 (1 er s. av. J.-C.) ; H. Menzel, Anlike Lampen im röm.-germ. Zentralmuseum zu Mainz, 1954, p. $23, n^{\circ} 71$.

(11) N. Lamboglia, Rev. Et. ligures, 1952, p. 210, fig. 64. 
Bronze. - Les objets de bronze sont très corrodés, au point que le métal a disparu presque entièrement sous sa couche d'oxydation :

fragment de grande patère à marli plat (fig. 7), imitant la forme de l'assiette campanienne (forme 6 de Lamboglia). présentant sur le marli de petits trous circulaires pour

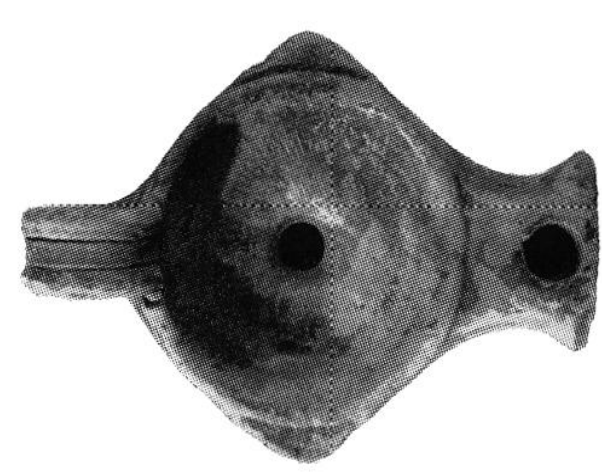

Fig. 4. - - Lampe à ailettes et anse annulaire, à couverte brun rouge. $1: 2$. Pholo $Y$. Rigoir.

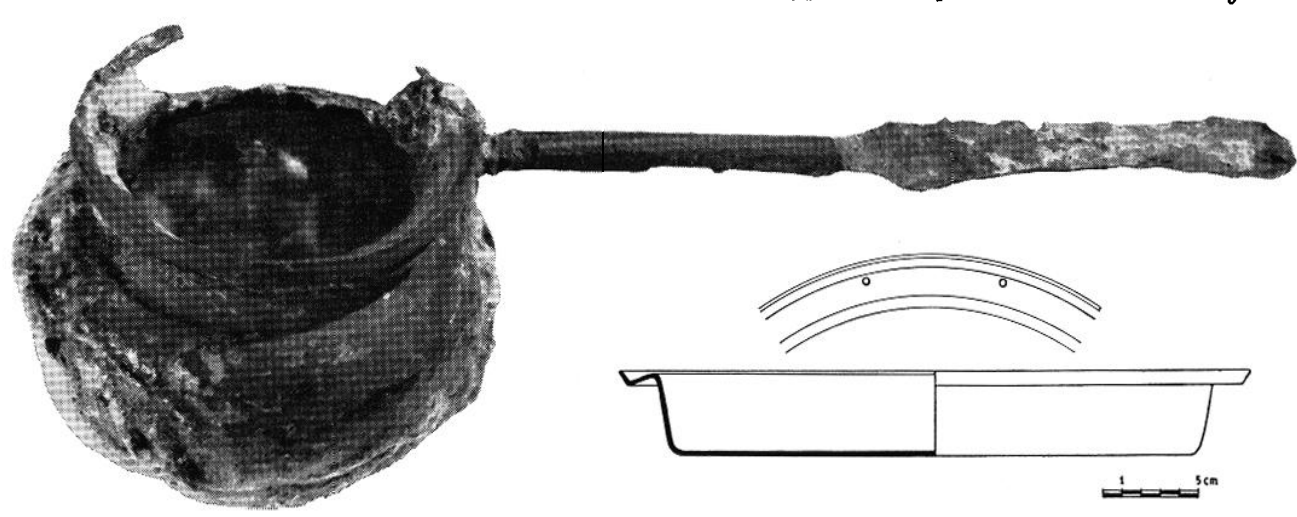

Fig. 6. - Cyathus de bronze, après nettoyage. Long. : 0,35. Photo Y. Rigoir.



Fig. 5. - Lampe à ailettes et anse annulaire, de même type. Face et profil. $1: 2$. Relevé Ch. Lagrand.



la fixation d'un couvercle (?) (diam. 0, 33 ; haut. 0, 041) ; cassolette à long manche (fig. 6), terminé par une queue plate (cyathus), enrobée dans une gangue de calcaire qui avait fait disparaître la paroi du récipient ; celle-ci a été reconstituée d'après le moulage de la gangue au Laboratoire du Musée Borély (long. avec le manche 0,35 , haut du récipient : 0,10 ); hameçons à pointe barbelée, d'un lype analogue à ceux qui ont été trouvés 
au Grand Congloué et à Nemi ${ }^{12}$; petite cuiller en bronze (long. 0,125) (fig. 8) ; feuille de bronze très mince en forme de moule, etc.

Plomb. - La coque du bateau n'avait pas de doublage de plomb, contrairement au mode de construclion des navires de commerce du Crand Congloué et d'Albenga et des navires de plaisance du lac de Nemi. Mais le plomb était utilisé dans le gréement : tuyau de plomb de 0,05 de diamètre, analogue à ceux qui ont été trouvés au Grand Congloué et à Albenga ; " pot » de plomb, de forme hémisphérique à l'intéricur, muni d'un anneau de fixation; le rebord porte quatre trous carrés pour le passage d'une corde, ainsi que le montrent deux pots analogues, sans anneaux de fixation, trouvés dans

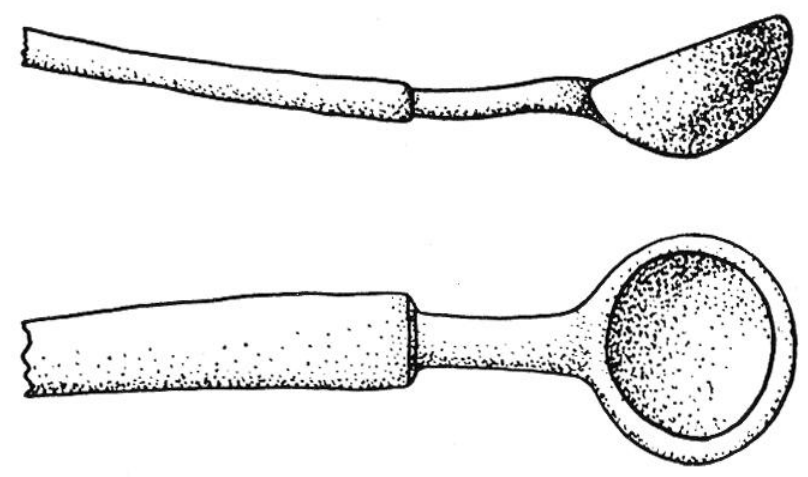

Fig. 8. - Cuiller en bronze. Long. : 0,125. 2: 3. Relevé Ch. Lagrand.

l'épave du grand Congloué, d'un usage indéterminé (plomb de sonde?) ; diverses lamelles de plomb et une bande de plomb de 5 millimètres d'épaisseur en fer à cheval, de 0,086 de diamètre, percée de deux trous ronds aux extrémités.

Divers. - Meule plate à farine, pierre à aiguiser, deux petites tablettes à arêtes biseautécs du type des cachels d'oculiste, l'une en schiste, l'autre en pierre dure, sans inscription, etc.

Bois. - L'intérêt capital de cette fouille, qui peut être datée, est la remontée particlle de la carène du batcau avec sa quille ct son bordage qui ont été déposés à la Tour Royale, dépendant du Musée de la Marine à Toulon. Les assemblages ont été reconstitués et relevés par $M$. Ch. Lagrand, stagiaire de recherches au C. N. R. S., sur les indications du Gt Tailliez, qui en avait assuré le sauvetage et l'émersion et interprétés d'après les avis techniques de M. Mauric, architecte naval.

La quille avec ses membrures était apparuc après dévasage, étalée sur le fonds sableux sur 20 mètres de longueur, comme le squelette d'un poisson gigantesque. On a pu remonter une section de quille de 10 mètres de longueur, avec la partie attenante des membrures correspondant au fonds, seul conservé.

(12) G. Uablli, Le navi di Nemi, 1950, p. 135, fig. 143. 
Cet assemblage, qui comporte une quille et une contre-quille ou carlingue, est tout différent de celui du bateau du Grand Congloué à Marseille, dont une petite section de carène a été remontée cette annéc même et de celui de Mahdia ${ }^{13}$, qui a été laissé au fond de la mer, par 39 mètres de profondeur.

Le bateau à fond plat, sans doublage de plomb, se rapproche au contraire de la "galère de César " ${ }^{14}$, conservée dans la vase et découverte en 1864 à

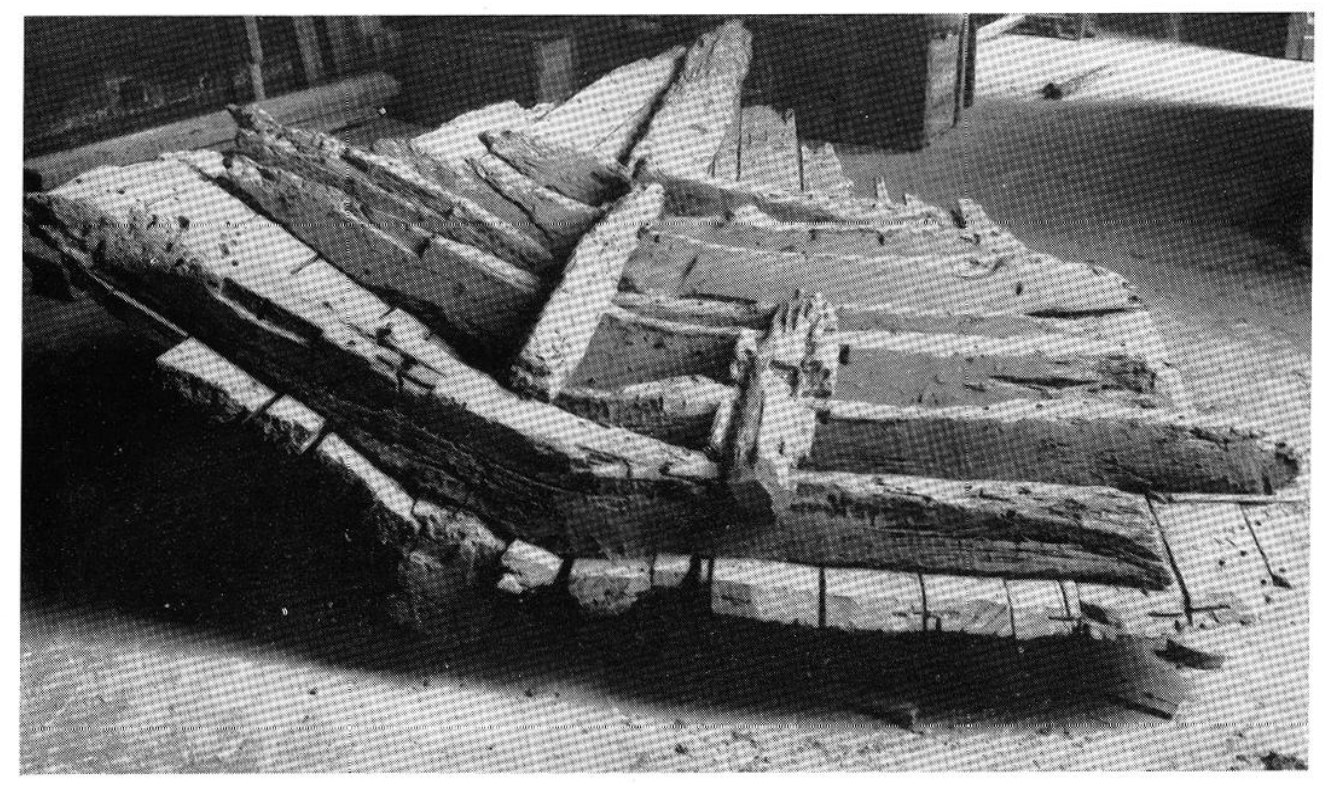

Figr. 9. - Bateau de commerce à fond plat, exhumé de l'ancien port, à Marseille en 1864. Vue de la section transversalc montrant la quille, le bordé extérieur attenant aux membrures et un segment de la carlingue, avec partie de virure du bordage interne (à droite). (Borély 16/1516).

Marscille (fig. 9), lors de la construction de l'immeuble portant le $n^{0} 3$ de la rue Impériale (aujourd'hui rue de la République), en un point qui correspond au fond du port à l'époque romainc. Les bois (pin d'Alep) de ce bateau de commerce, à fond plat, présentant des assemblages mortaisés et un cloutage de cuivre analogue à ccux du Grand Congloué et du Titan, sont conservés au Musée Borély, malheureusement démontés lors des transformations du Château en 1937. Une

(13) Guy di Frondeville, Les Visiteurs de la mer, Paris, 1956, p. 221, fig. 7.

(14) W. Froenner, Musée de Marseille. Catalogue des antiquilés grecques et romaines, 1897, n 16/1516; M. Clese, Massalia, II, 1929, p. 173, fig. 2 (photo de la partie médiane du bateau, improprement appelée "proue n) et p. 174. I.a maquelte d'Augier : Borćly 3747 et 3 clous de bronze : $n^{\circ} 879: 2379$. On ne peut se fier pour la date au contexte des céramiques qui auraient étć trouvées au niveau du batcau (Cat. 2045-2065, 3545-3565), la plupart des provenances indiquées par Augier étant fausses (F. Brxort, La constilution du Musée Borély, dans Provence historique, VI, 1956, p. 9). 
maquette d'H. Augier, reproduisant cette partic de bateau " avec une exactitude mathématique " (Froehner), permettra d'en faire la reconstitution.

La comparaison de la section de la quille de ces quatre épaves est intéressante par elle-même, indépendamment des différences de construction des quatre carènes; mais elle ne peut donner de base de comparaison, l'épaisseur de la quille variant selon le point où a été faite la section, plus forte au centre qu'à l'une des extrémités : la quille du Titan, correspondant sans doute au milieu

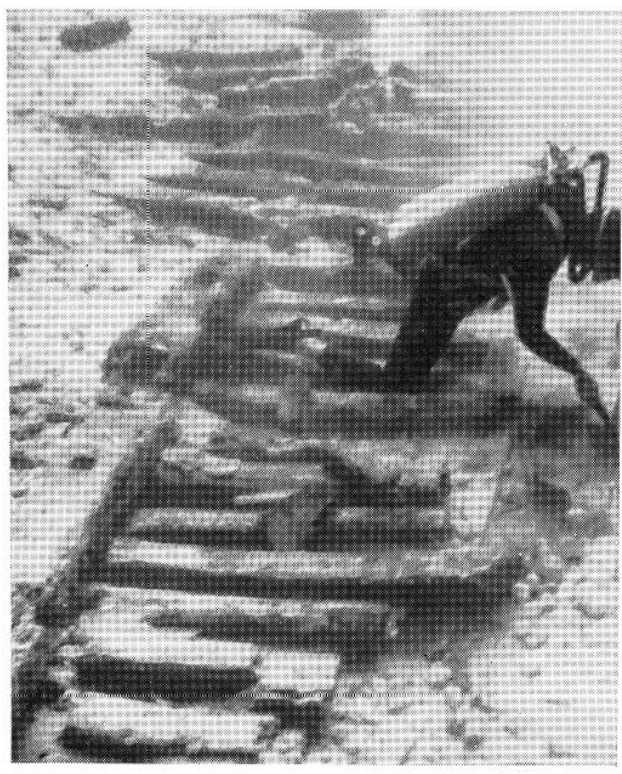

Fig. 10. - Épave du Titan. Le fond de la carène de l'épave après dévasage. Dans l'axe longitudinal la carlingue, percée de deux trous pour les chevilles d'assemblage de la quille (fig. 13, 7) avec les membrures et le bordage extérieur. Pholo $C^{\text {t }}$ Tailliez.

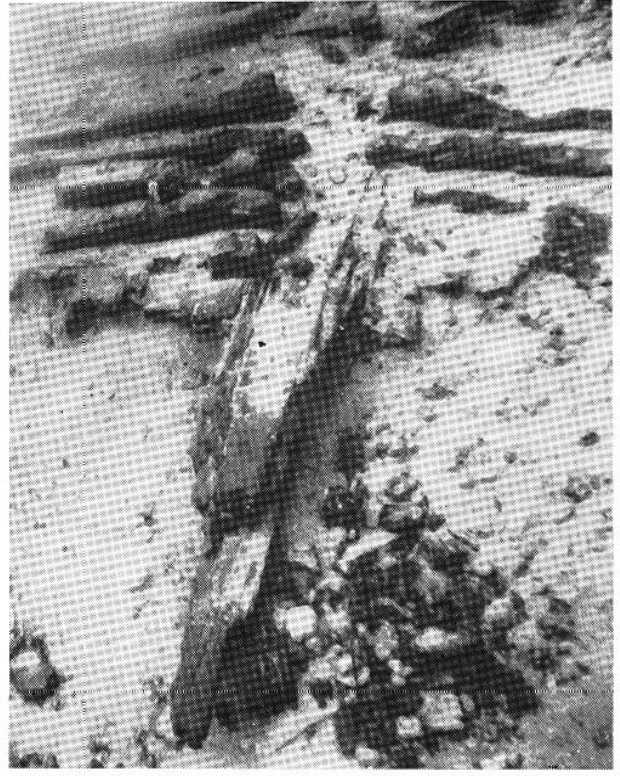

Fig. 11. - La quille avec les membrures, après enlèvement de la carlingue ou contre-quille. Phoio $C^{\text {t }}$ Tailliez.

de la carène (haut. 0,22 ; larg. 0,195), est intermédiaire entre celle de Mahdia appartenant sans doute à la poupe ou à l'étambot, en raison de la forme de la carène (haut. 0,29; larg. 0,23 ; en une autre partie 0,20 environ de côté) ${ }^{15}$, et cclle du Grand Congloué correspondant à la partic extrême du bateau (haut. 0,17 ; larg. 0,12 ), qui serait de plus faible tonnage. Si le premier portait en poids un chargement de marbres de 200 à 230 tonnes $^{16}$, les autres avaient sans doute un tonnage plus faible, qui ne dépassait pas cent tonnes de porids.

(15) G. de Frondevilue, o. c., p. 220.

(16) C'est approximativement le poids que portait le bateau de marbres de Carrare échoué à Saint-Trope\% : Rev. El. ligures, 18, 1952, p. 244. 
Des comparaisons utiles peuvent également être faites avec les bois du bateau de New Council Hall, du III ${ }^{\mathbf{e}}$ siècle de notre ère, découvert à Londres en $1910^{17}$ et aussi avec les navires retrouvés au lac de Nemi, qui ont péri dans un incendic en 1944, mais dont les relevés ont été conservés ${ }^{18}$.

Les grosses pièces de bois, constituant la charpente du bateau, sont en pin (quille), en chêne et peut-être en cyprès.

Ce qui caractérise ce navire, un bateau à fond plat, c'est que la quille est doublée par une carlingue, dans l'axe longitudinal du bateau (fig. 10-11 et relevés 13-14); elles étaient liées l'une à l'autre, à des distances variables, par

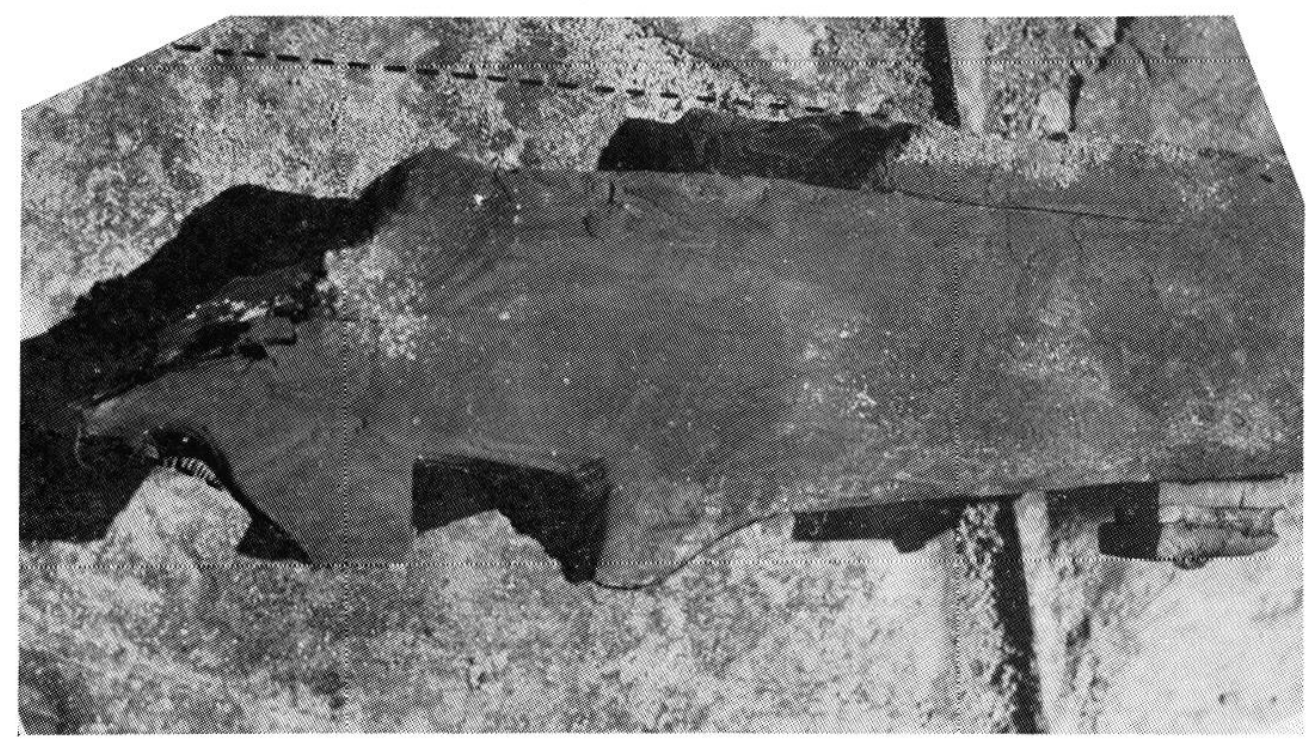

Fig. 12. - Partie médiane de la varangue d'une membrure avec le trou d'anguiller (fig. 13, 8). Au-dessous partie de bordé avec tenon d'assemblage. Photo $C^{*}$ Tailliez.

des pièces de bois verticales, grosses chevilles, qui traversaient les membrures, afin d'assurer la rigidité de la carène dans le sens longitudinal. Les membrures, longues traverses perpendiculaires à cet axe, sont d'un seul tenant, comme dans le bateau de 1864 ; la partic la plus épaisse, la "varangue ", s'insère dans des entailles ménagées sur la tranche inférieure de la carlingue; les membrures très rapprochées sont distantes de 0,15 cnviron l'unc de l'autre ; une réparation sur un côté de la carène avait fait insérer entre deux membrures une pièce supplémentaire.

(17) W. E. Riley et G. L. Gomme, Ship of the Roman Period discovered on the New County IIall Sile (The London County Council), Londres, 1910 ; plan reproduit par G. Ucril.1, o. c. (note 4), p. 251, fig. 283.

(18) G. Ucelli, Le navi di Nemi, pl. I-X. 


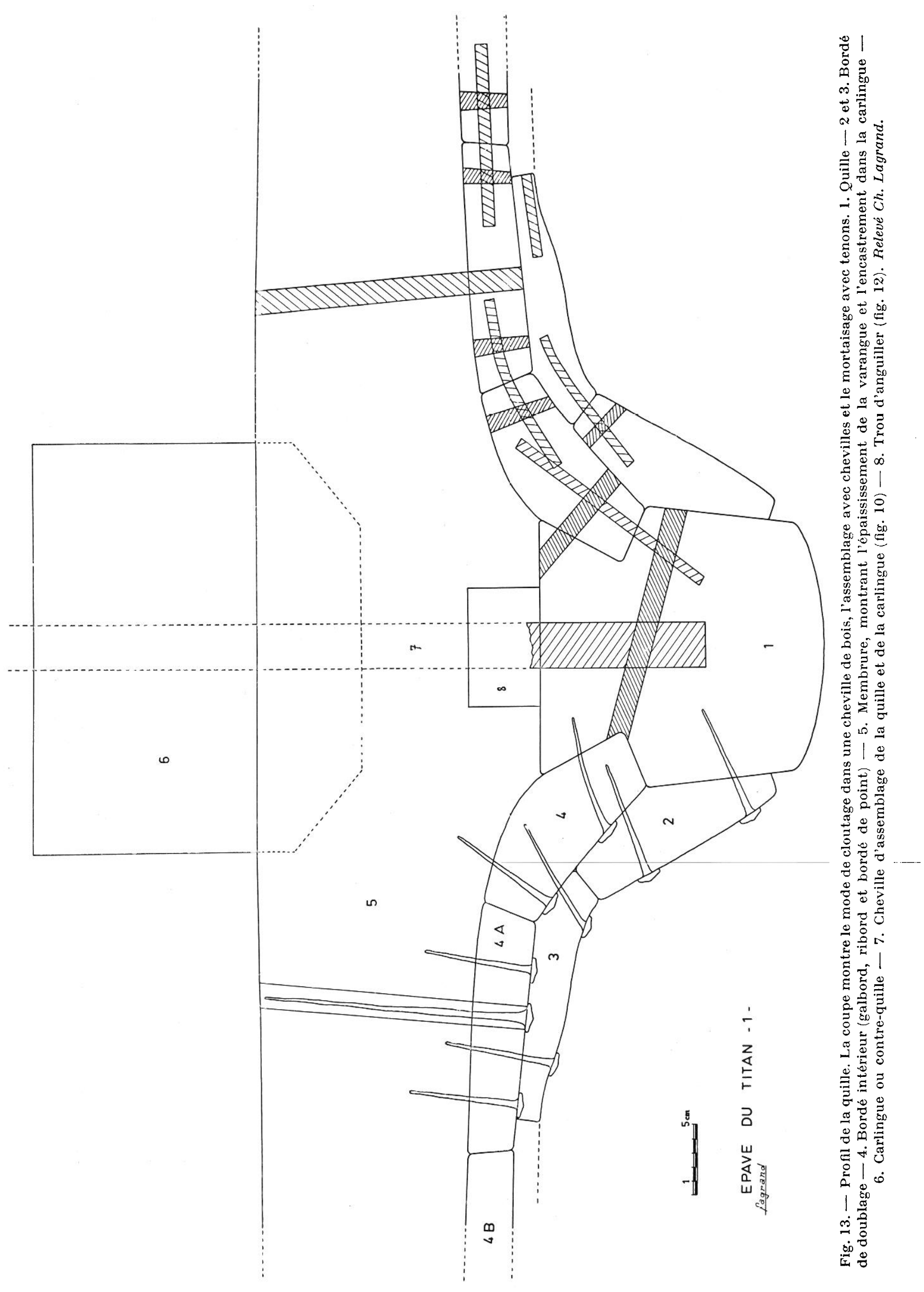




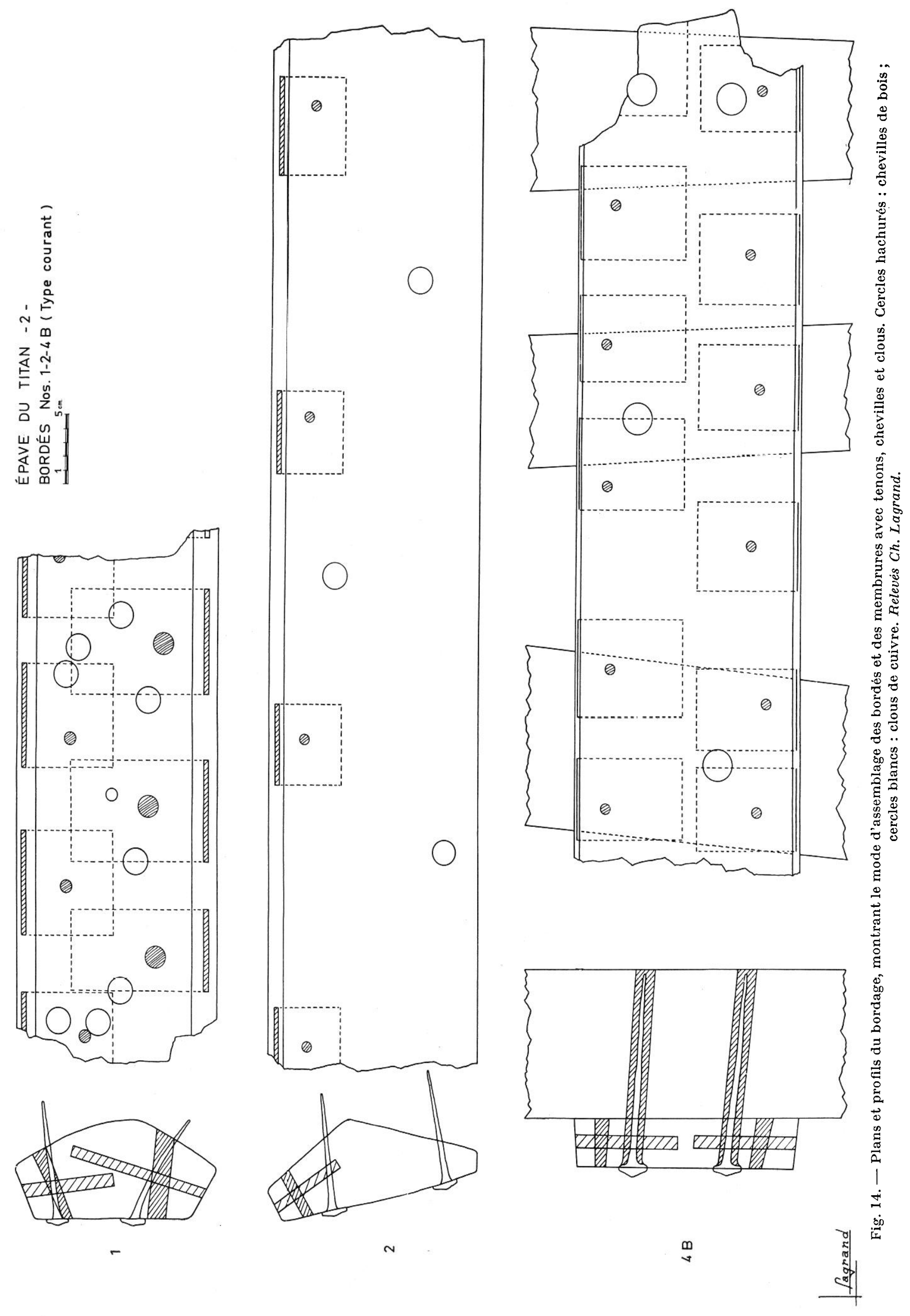


Cette disposition est toute différente de l'assemblage de la carène du Grand Congloué : les membrures, indépendantes des deux côtés de la quille, sont isolées de celle-ci ; mais elles sont renforcées par d'épaisses varangues de forme triangulaire, indépendantes, liées aux membrures et au bordé par des clous, destinćes à maintenir constant l'écartement des deux branches des membrures et à assurer la rigidité de la carène dans le sens transversal. Il semble que l'assemblage du bateau de Mahdia soit conçu selon ce typc.

L'écoulement de l'eau se faisait par des trous d'anguiller percés au bas de la varangue, au centre de chaque membrure (fig. 12), selon un dispositif analogue à celui du Grand Congloué, dont les pièces de varangue superposées aux membrures étaient percées d'un trou triangulaire ${ }^{19}$.

Lc profil du balcau ne peut malheureusement être restitué, les membrures n'étant conservées que sur une longueur d'1 m. 50 à 2 mètres de chaque côté de la quille, c'est-à-dire dans la partie qui correspond à l'empâture, sectionnéc avant la courbure de la branche, qui aurait donné la largeur de la carène. Seule une membrure comporte à 2 mètres de la quille le début d'une courbure, ce qui donnerait une largeur de 4 mètres au fond de la carène.

Cette partic du bateau, quille, carlingue et membrures, correspond à la grosse charpente. Il reste peu de chose du bordage extérieur (fig. 10) et rien du revêtement de bois intérieur ou vaigrage, dont les virures longitudinales étaient appliquées sur le côté intérieur de la membrure.

Le bordage extérieur est constitué par des madriers de peu d'épaisseur $(0,04)$, correspondant au fond de la carène. Il est, réduit aux pièces qui assurent la jonction avec la quille, le galbord, le ribord et les deux premières traverses du bordé de point. Il semble, au moins dans la partie la plus voisine de la quille, que cc bordé ait été doublé par un bordé extérieur (fig. 13), que l'on retrouve également dans la section de carène remontée au Grand Congloué, laquelle était plaquéc de plomb.

Ces pièces étaient solidement assemblées par des tenons et des mortaises, par des chevilles de bois et par des chevilles de chêne traversées par de grands clous de cuivre tordus à lcur extrémité, sur le type des assemblages du Grand Congloué et d'Albenga.

La section du bordage, plus épaisse vers la quille, analogue à celle du Grand Congloué, est conforme à ce que nous savons de la construction des navires antiques, dont lc bordé ne dépassait pas 6 à 8 centimètres d'épaisseur ${ }^{20}$.

(19) Dans le bateau de Londres, plus tardif et d'une exécution sommaire, l'écoulement de l'cau se faisait par plusicurs petits anguillers ménagés au bas des membrures (RII.EY-Gomml, o. c., p. 9, fig.).

(20) Cecil Tork. s. v. navis, dans Dict. Antiq., IV, p. 32, d'après Drog. Laerce, I, 103 ; Dion Curysostomf, Oralio 61 ; Juvénal., Sat., XII, 58, 59. 
Dans la marine antique et dans la construction des vaisseaux à voile du $\mathrm{XVIII}^{\mathrm{e}}$ siècle, la faiblesse des bordés était compensée par une armature longitudinale à l'extérieur, les préceintes ou traverses courant horizontalement le long du bateau, et à l'intérieur par des ceintures longitudinales ou bauquières : aucun témoin de ces pièces de renforcement n'a été trouvé ni à Mahdia, ni au Grand Congloué, ni au Titan, ce qui laisse en suspens la question du pont qui était soutenu par les bauquières.

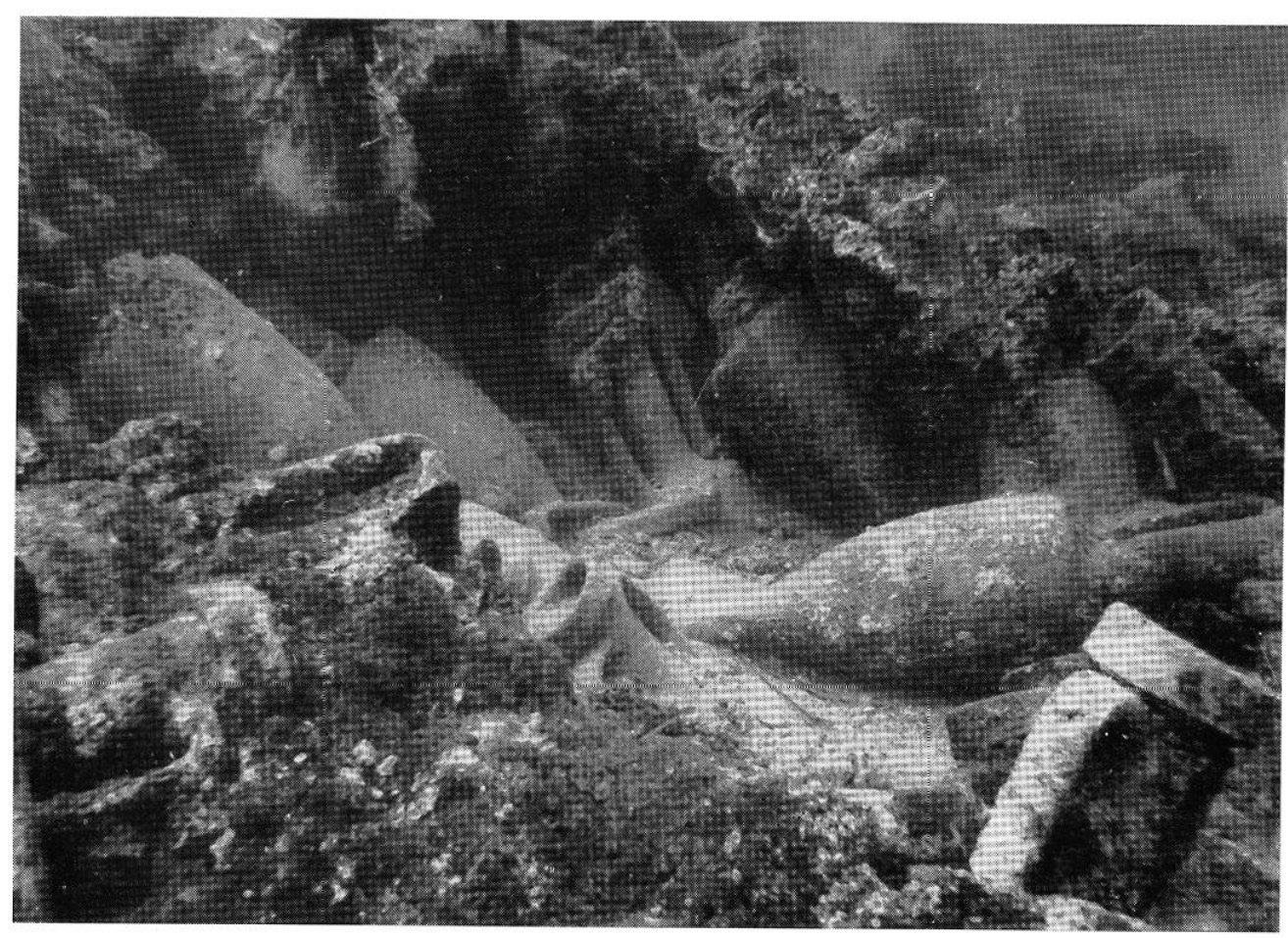

Fig. 15. - Epave du Dramont. Amphores dans leur lit de pose (incliné) sous la gangue de concrélions marines. Photo J. Auberlet.

Les observations faites à Mahdia ne donnent au reste aucune certitude sur l'architecture des parties hautes du navire, au-dessus de la ligne de flottaison, ni sur l'existence d'un pont continu ou de petits ponts à l'avant ou à l'arrière ; la présence de tuiles dans les divers vaisseaux de commerce chargés d'amphores, relevés en Méditerranée, au Grand Congloué, à Albenga, au Dramont, font supposer qu'en poupe se dressait une construction légère (camara) correspondant au " gaillard d'arrière ".

2. Épave du Dramont. - L'épave découverte par M. Claude Santamaria, prospectée par le "Groupe de plongée sous-marine" de Saint-Raphaël 
( $\mathrm{D}^{\mathrm{r}}$ Delonca, J. Auberlet, etc.) à 180 mètres S.-O. de l'Ile d'Or, par 35 mètres de fond, dans la baic de Saint-Raphaël a un grand intérêt par la diversité de ses amphores et de ses marques amphoriques.

L.e naufrage a été provoqué par un écucil, dont le sommet est à $2 \mathrm{~m} .50$ environ sous lc niveau de la mer, le navire gisant au pied du tombant : c'est là un principe constant dans l'archéologie sous-marine, qui a déjà été noté aux balises do la Chrétienne et du Titan, à la Louve du Cap Gros à Antibes, etc., dont les récifs étaicnt d'autant plus dangereux qu'ils affleuraient la surface de la mer.

Le chargement formant un "tumulus » de 21 mètres sur 8 environ, est enrobé dans une gangue de concrétions calcaires qui alteste l'homogénéité de l'épave : cette gangue, qui a dû être attaquéc à coup de pic l'avait protégé, jusqu'à sa découverte, malheureusement suivie de pillages el de dynamitage au cours de l'été 1957. Sous cette gan-



Fig. 16. - Dramont. Amphores porlant la triple marque de Pilip(us) (Borély C. 27) et la marque d'IIeraclius (C. 20), avec le contre-sceau S suivi de l'ancre sur le col. (v. ljg. 17-18). -.. 1 : 10. Relevé Ch. Lagrand. gue, les amphores apparaissent dans leur lit de pose, légèrement inclinées, mais intercalées, les pointes du rang supéricur reposant sur les épaules de la couche inféricure (fig. 15). Un tel chargement vertical comprenait donc plusieurs lits d'amphores superposées, qui devaient être protégécs par des "paillons".

Ces amphores appartiennent à plusicurs types dont le synchronisme pose une 
én gme. Le groupe le plus nombreux, dont une vingtaine d'échantillons ont été remontés, appartient, à la forme la plus récente et la plus volumineuse (haut : $1 \mathrm{~m} .15-1 \mathrm{~m} .26$ ) du type Dressel I, qu'il y a lieu de différencier par un indice B du groupe de lradition plus ancienne (Dressel I $\Lambda$ ) dont la hauteur ne dépasse pas 1 mètre ${ }^{21}$. I'amphore cylindrique, à pied massif se raccordant à l'ogive de la panse est remarquable par le profil de la lèvre, verticale ou peu inclinée, parfois concave dans les exemplaires les plus hauts; elle atteint $0 \mathrm{~m} .06$ à $0 \mathrm{~m} .08$ de hauteur.

On peut distinguer dans ce groupe deux variétés : l'amphore la plus haute $(1 \mathrm{~m} .20$ 26), ayant une lèvre de $0 \mathrm{~m} .08$, ne porte pas de marque ; celle-ci parait être réservée à la variété un peu moins haute, de $1 \mathrm{~m}$. 15-18 (fig. 16). Une dizaine de ces dernières comportent en effet des marques doubles, imprimées sur la lèvre et sur l'épaule, près de l'altache de l'anse et dans un cas au bas du col, parfois suivies d'une contremarque sur la lèvre. Ces marques sont diflérentes, comme celles de l'épave de la Ciotat.

Les noms inscrits dans un cachet rectangulaire ( 0,030 sur 0,018 environ) (fig. 17-18) apparticnnent à des gentilices connus en Campanie ${ }^{22}$, dont plusieurs se retrouvent en Narbonnaise : $\operatorname{BAC}($ chius $), \operatorname{DAM}(a)^{23}, \operatorname{EVTA}(\text { clus })^{24}$,



Or, la même amphore associe parfois deux noms différents, l'un sur la lèvre, l'autre sur l'épaule, avec alternance d'emplacement et d'accouplement ; PILIP est reproduit trois fois sur la même amphore, sur la lèvre, au bas du col et sur l'épaule (fig. 16,1), mais il est sur d'autres exemplaires associé à DAM et à IIERM, tandis que BAC l'est à EVTA et à ONEL (fig. 18).

Il est peu vraiscmblable en ce cas de voir dans l'un des deux noms celui du potier ; et l'on peut supposer que l'un et l'autre nom désignent des producteurs associés, peut-être parents, qui ont tenu à faire figurer leur double marque sur l'amphore à un emplacement indéterminé.

Mais Ies six premiers timbres sont suivis, sur la lèvre seulement, d'une contremarque carrée (16 millimètres de côté) portant un $\mathrm{S}$ suivi d'une ancre sans jas (fig. 18), aux pattes largement ouvertes comme le signe de l'ancre dans les marques cnidiennes trouvées à Délos ${ }^{26}$.

Si les navires de La Ciotat et du Dramont contiennent chacun des amphores de même type, mais de timbres variés, il n'y a aucune raison de voir dans ces

(21) Cr. N. I.amboglia, Sulla Cronologia delle anfore romane di elà repubblicana dans Rev. Et. ligures, 21, 1955, p. 247, fig. 3 et 4 ; et F. Bevort, Typologie el épigraphie amphoriques, ibid., 23, 1957.

(22) Bacchius: CIL, X, 7373 (IIimera); Dama, 867, 924 (Pompei), 1403 (IIerculanum), 6397 (Terracine) ; Eutactus, 6406 (Terracine) ; IIeraclius, 2829, 3403 (Misène); IIerma, 1094 (Nocera), 3021 (Pouzzoles).

(23) Marque à Vienne : XII, 5683, 73.

(24) Marque à Toulouse (signaculum): ibid., 5690, 44.

(25) Marque à Narbonne, sur col d'amphore : ibid. 5683, 219.

(26) V. Grace, Timbres amphoriques trouvés à Délos, dans Bull. Corresp. hellénique, 76, 1952, p. 533, n $^{\circ} 4$ et pl. 19,4 . 


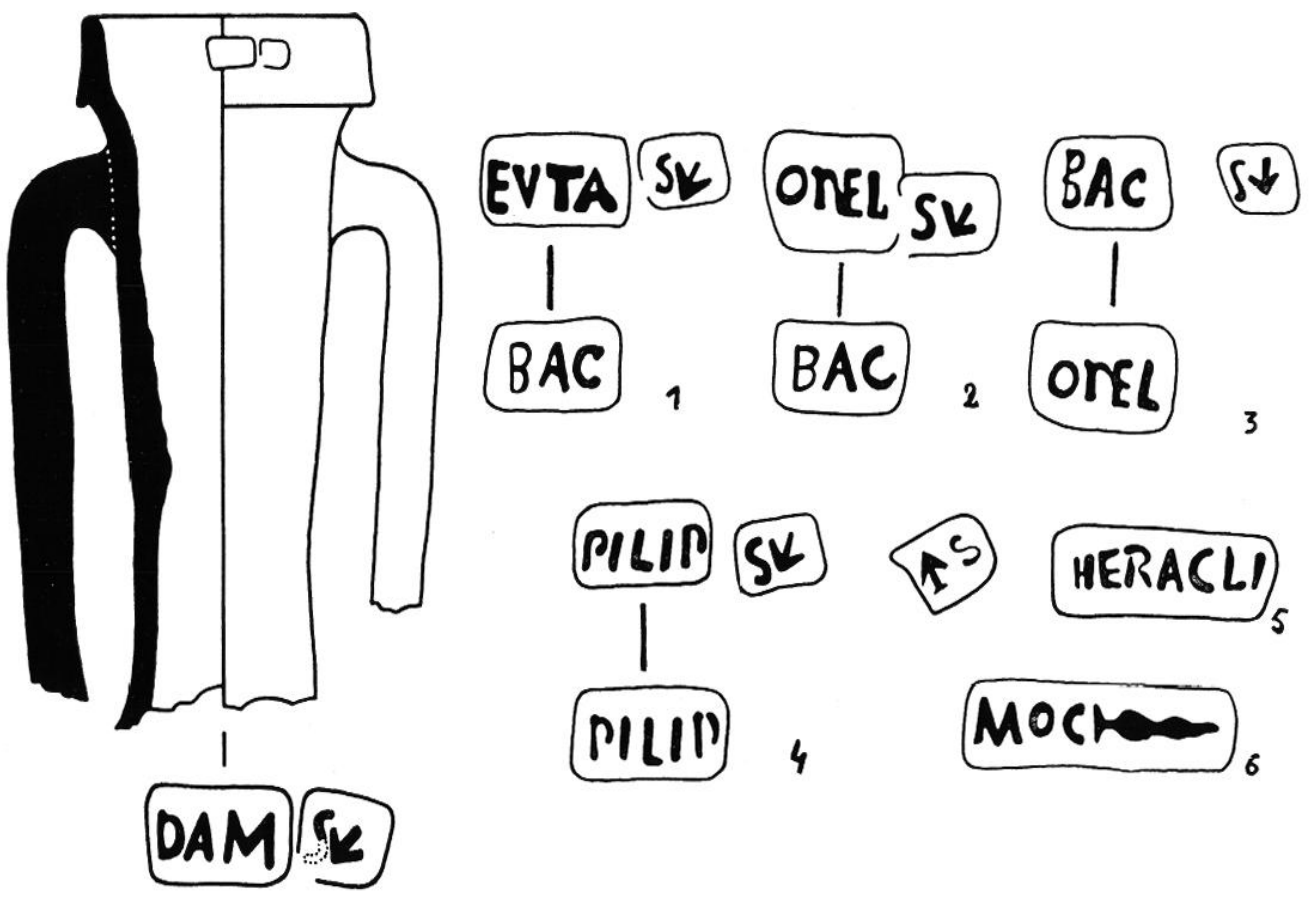

Fig. 17. - Dramont. Col d'amphore portant la marque Dam (a) aveçle contre-sceau (Borély C. 28). $1: 6$. Relevé Ch. Lagrand.

Fig. 18. -- Dramont. Estampilles des amphores : 1 à 4 : en haut, timbre du col (marque et contre-sceau); en bas, timbre de l'épaule; -5 et $6:$ timbres du col. $1: 2$. Relevé Ch. Lagrand.


Fig. 19-21. - Dramont. Amphore à huile - Amphore à col en bourrelet et anses bifides - Amphore à olive de "type punique " avec son bouchon de terre cuite. $1: 10$. Relevé Ch. Lagrand. 
noms celui du poticr ou bien du transporteur. Mais la contremarque $\mathrm{S}$ suivie de l'ancre sur la lèvre de plusieurs amphores du Dramont, qui groupent la récolte de plusieurs producteurs, a un sens différent, auquel le symbole de l'ancre donne une valeur maritime; son indépendance du timbre au nom du producteur montre qu'il est différent de celui-ci, - ce qui n'est pas le cas pour le Sestius de l'épave du Grand Congloué, dont le symbole accompagne le nom sur le même cachet ${ }^{27}$.

Ce cachet ne serait-il pas la contremarque du négociant qui aurait acheté à la sortic du pressoir la récolte de plusieurs propriétaires de la région et fait préparer à la poterie leur " emballage » pour l'écouler sur le marché de la Gaule ?

Il a été également remonté de cette épave plusieurs autres lypes d'amphores qui sont synchroniques : une amphore pansue à huile (fig. 19) à col mouluré ct lèvre en quart de rond ${ }^{28}$, une amphore à panse de forme ovoïde de plus d'un mètre de haut, à col tronconique court retréci vers le haut et lèvre en ourlet avec anses bifides coudées à angle droit (fig. 20), du type tardif de $\operatorname{Cos}^{29}$ et une amphore du type dit punique (haut: $0 \mathrm{~m} .785$; diam. $0 \mathrm{~m}$. 185) munie de son opercule de fermelure (fig. 21) et encore remplie d'olives, au milieu desquelles étaient des brindilles de tiges. Ce type d'amphore, issu de l'amphore phénicienne et punique, dont la fabrication ne saurait être recherchée ailleurs que dans le Sud de l'Italie, est maintenant connu par l'ćvolution typologique qu'en a tracée Virginia Grace, avec le matériel des fouilles de l'agora d'Athènes ${ }^{30}$. L'exemplaire le plus ancien en Provence a élé trouvé dans les fouilles de l'oppidum des Pennes du III $^{\mathrm{e}-\mathrm{II}} \mathrm{e}^{\mathrm{e}} \mathrm{s}$ (musée Borély 11719 ) ${ }^{31}$; il mesure $0 \mathrm{~m} .87$ de haut et $0 \mathrm{~m} .22$ de diamètre ; le corps cylindrique muni de deux petites anses au sommet de la panse, à col court terminé par un rebord droit, se réfère à l'amphore S S 14069 de l'agora, datée de 200 par V. Grace G2 $^{32}$.

On en rapprochera les amphores à collerette droite, remployées en tuyauterie, trouvées dans les fouilles terrestres de Ruscino $0^{33}$, près de Perpignan, qui ont une origine ibéro-punique et remonteraient au $\mathrm{III}^{\mathrm{e}}-\mathrm{II}^{\mathrm{e}}$ siècle.

Les amphores provenant des fouilles sous-marines malgré leur référence à ces types sont de date moins ancienne. Les amphores de Fos, découvertes récemment dans l'anse Saint-Gervais (haut. $0 \mathrm{~m} .785$ et $0 \mathrm{~m} .70$; diam. $0 \mathrm{~m} .23$ et 20) sont à rapprocher des amphores S 14069 et P 6762 de l'Agora à collerette droite et biseautée, datées de 200

(27) Gallia, XII-1954, p. 44, fig. 5.

(28) Dressel 10 (Lamboglia, Sulla cronologia type 7).

(29) Type voisin de Dressel 5 (Lamboglia type 5); voir Gallia, XIV, 1956, p. 24, fig. 1, no 6-10; et F. BENOIT, Typologie el épigraphie amphoriques, $l . c$.

(30) The Canaanite Jar, dans The REgean and the near East, Sludies presented to Helty Goldman (New York), 1956, p. 80-109.

(31) G. Vasseur-Chaillan, L'oppidum de la Teste Nègre aux Pennes, dans Ann. Fac. Sciences de Marseille, 24, 2, 1917, pl. 1, 4 et 5; du même site provient une amphore pansue sans ogive terminale, terminée par un mamelon (pl. I, fig. 1), que V. Grace date du Ive s. d'après l'exemplaire d'Ampurias (l. c., fig. $\left.\mathrm{n}^{\circ} 2\right)$.

(32) L. c., p. 89, fig. 6, no 3 .

(33) Fouilles de G. Claustres, dans Gallia, XIV-1956, p. 204, fig. 1. 
et de la deuxième moitié du II $^{\mathrm{e}}$ siècle ${ }^{34}$; elles ne peuvent remonter au-delà de la création du port de Fos par Marius (104 av. J.-G.). L'amphore du Dramont à collerette moulurée se rattache aux amphores de l'agora S S 6598 et 6815 (haut. : 0 m. 78 ; diam. : 0 m. 21-19) de la fin du rie $\mathrm{s}^{35}$. Son association à l'amphore italique Dressel 1 et à l'amphore à anses bifides Dressel 5 incline à l'abaisser à la fin de la première moitié du err $^{\mathrm{s}} \mathrm{s}$, selon l'évolution lypologique qui peut être précisée par les épaves d'Albenga, de Gênes-Pegli et de la Maddalena en Sardaigne ${ }^{36}$.

La provenance de ces amphores pose un autre problème. Si le type se réfère à celui de l'amphore punique de $\mathrm{P}$. Cintas ${ }^{37}$, daté par les nécropoles des $\operatorname{IV}^{\mathrm{e}}-\mathrm{II}^{\mathrm{e}} \mathrm{s}$., auquel appartiennent les exemplaires de Ruscino, la fabrication de ces amphores
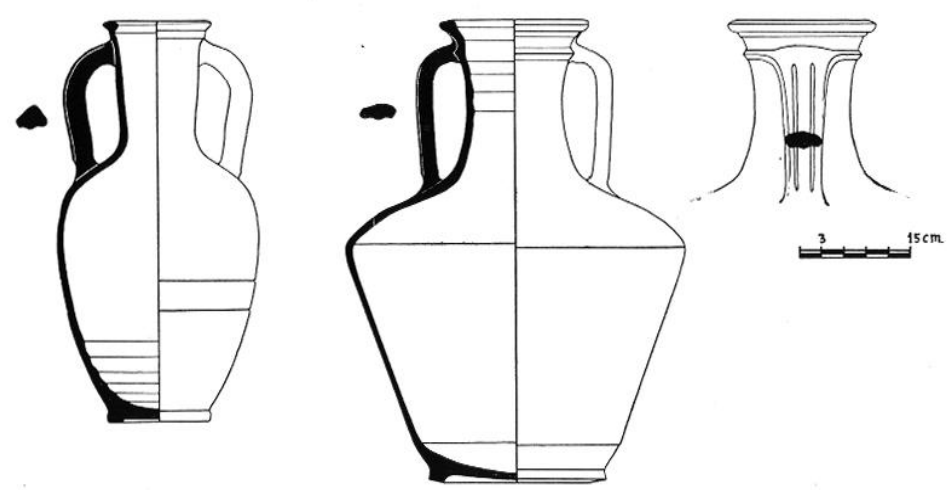

Fig. 22. - Dramont. Cruches à deux anses. Relevé Ch. Lagrand.

ne saurait être qu'italique : c'est ce que nous avions supposé pour les exemplaires d'amphores de type dit punique, à panse effiléc, de dimension moindre (haut. 0,82 ; diam. au haut de la panse 0,15 ), trouvées avec le lot des amphores de Sestius, au Grand Conglouééc, dont la référence à la datation de l'exemplaire (incomplet) de l'agora P. 8946, du début du I $^{\text {er }}$ siècle ${ }^{39}$, paraît par contre trop basse.

Cette épave contient en outre de très nombreux vases en poterie commune et de la vaisselle de cuisine en parfait état de conservation : cruches à une et dcux anses (fig. 22-25), dont une à deux anses de très grande taille $(0,80)$, marmites, etc.

(34) L. c., fig. 6 , nos 3 et 4 et infra, no 7 et fig. 45 .

(35) L. c. fig. 6 , nos 5 et 6.

(36) Sur ces épaves N. Lamboglia, Sulla cronologia, p. 247 et G. Rogir, Una nave romana con tulto il carico, dans L'Europeo (Milan), no 627,20 ost. 1957.

(37) Céramique punique, p. 149 et pl. $26, \mathrm{n}^{\circ} 312$ et 313 et pl. 94.

(38) Gallia, XIV-1954, p. 43 et fig. 2 (type VI).

(39) V. Grace, l.c., fig. $6, \mathrm{n}^{\circ} 7$. 


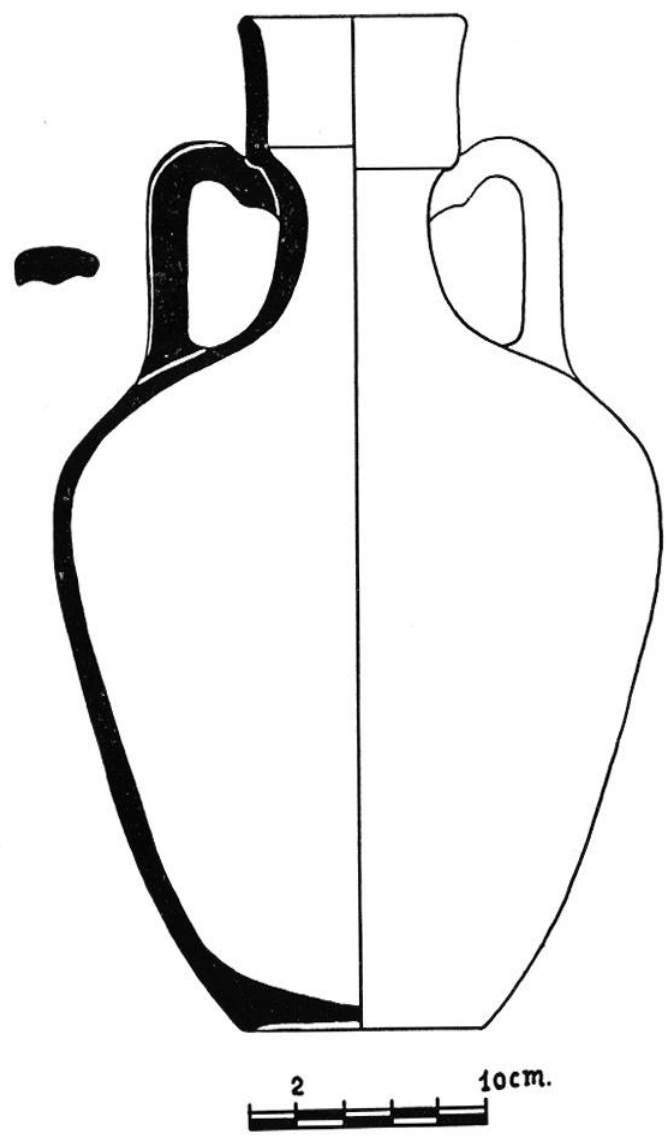

Fig. 23. - Iramont. Cruche à collerette, à deux anses. Relevé Ch. Lagrand.

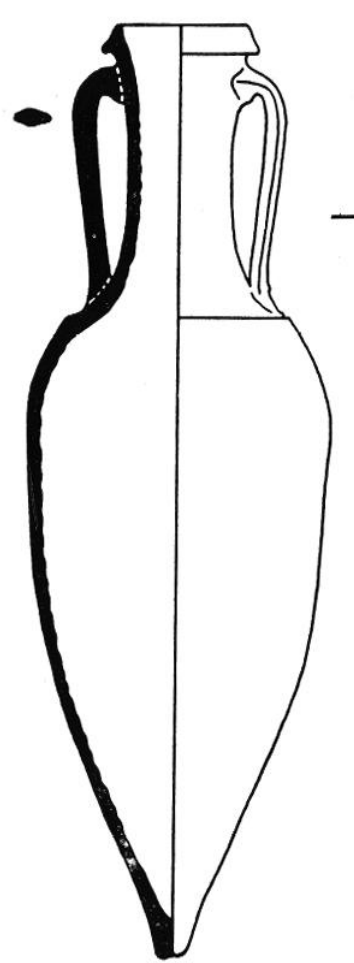

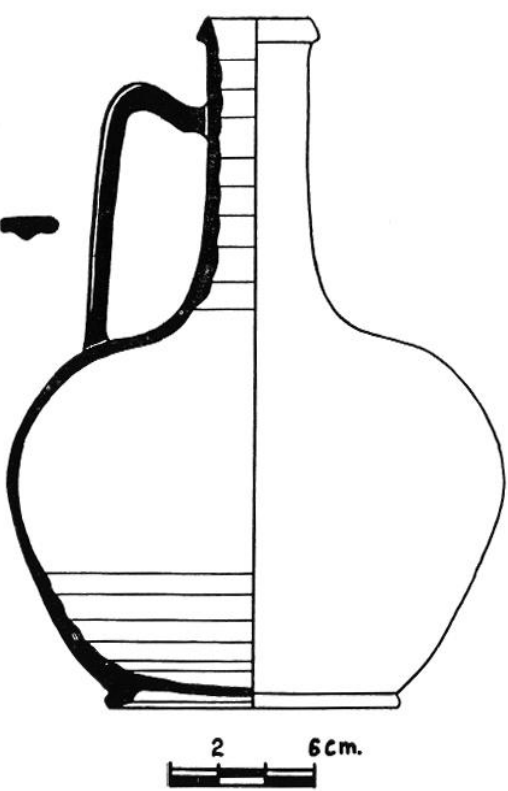



Fig. 24-25. - Dramont. Olpé à une anse. - Petite urne à deux anses. Relevé Ch. Lagrand.
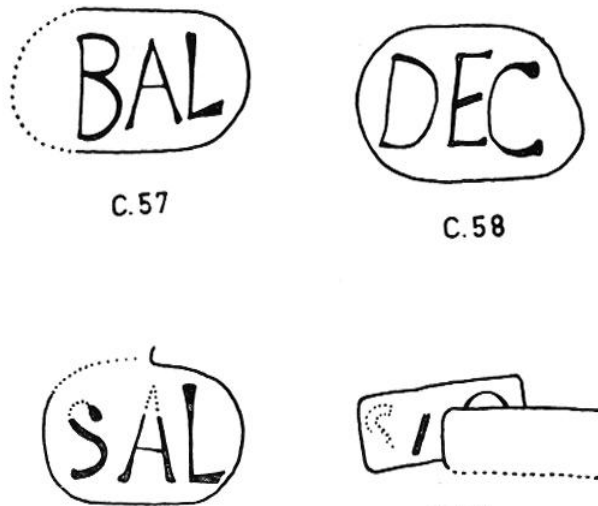

C. 59

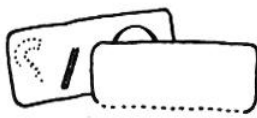

C. 60

Fig. 26. - La Ciotat. Amphore gréco-italique portant le timbre de Varus (?) (Borély 6776). $1: 10$. Relevé Ch. Lagrand.

Fig. 27. - La Ciotat. - Timbres Bal(bus), Dec(ember?), Sal(vius), (?) Salo, (Borély C. 57-60). 1. : 2. Relevé Ch. Lagrand. 
3. Épave de La Ciotat. - Découverte en 1956 par M. G. Bout et reconnue par H. Portail, cette épave se trouve entre l'Ile Verte et le Bec de l'Aigle, à 15 mètres de la Balise du Canonnier du Sud, entre 18 et 24 mètres de profondeur, dans la baie de La Ciotat.

De nombreuses amphores ont été retrouvées et quelques-unes ont pu être étudiées et déposées au Centre de documentation du Musée Borély. Ce type,

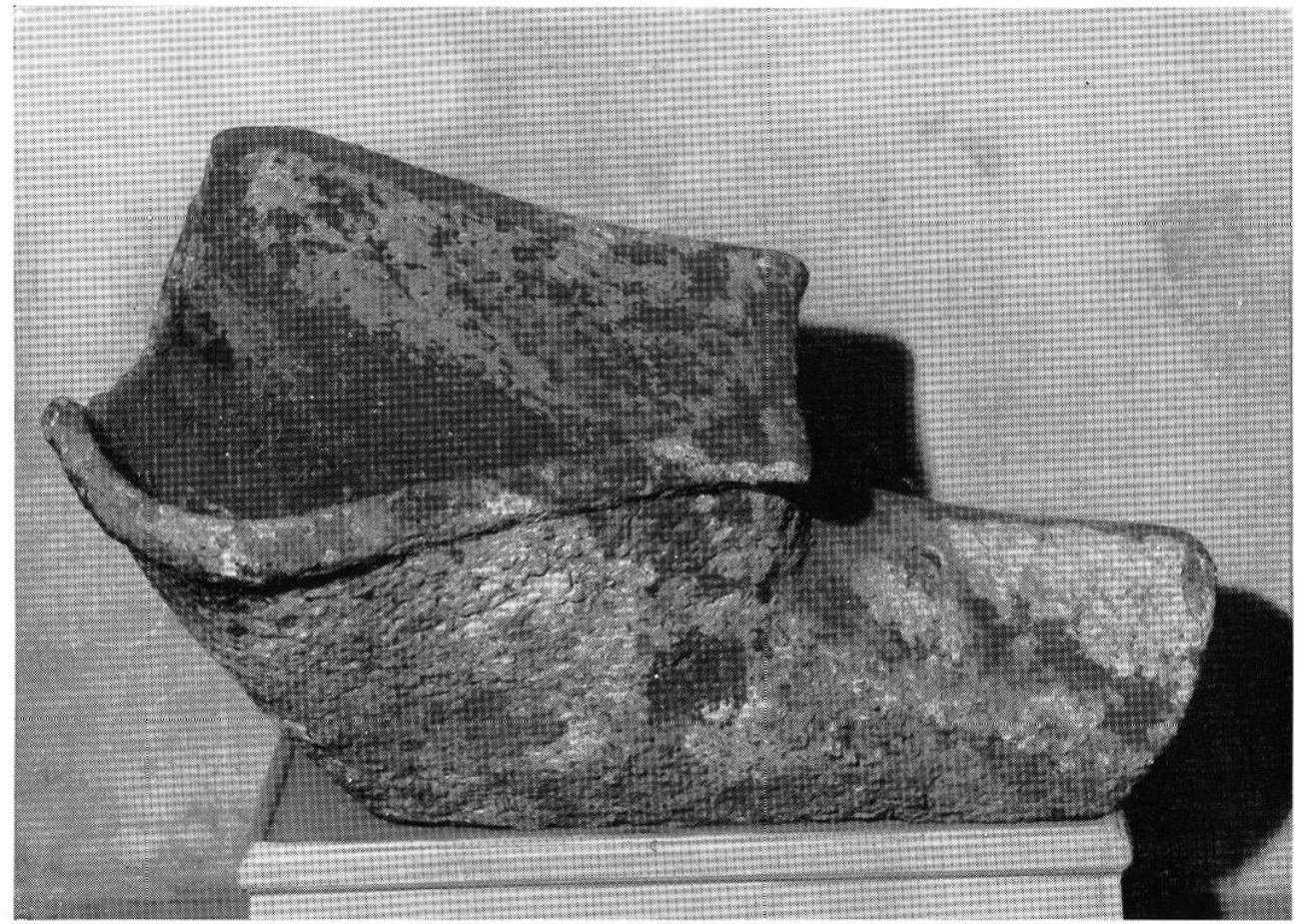

Fig. 28. - La Ciotat. Baignoire-sabot. Long. 0,90. Photo Y. Rigoir.

qui dérive de celui du Grand Congloué, appartient au monde hellénistique : il est inconnu de Dressel. L'amphore est caractérisée par sa forme en toupie, à épaulement, marqué par une arête, le col relativement court et la lèvre courte et inclinée (fig. 26). A la différence des amphores du Grand Congloué, l'attache des anses au haut du col est nettement détachéc de la lèvre. D'une hauteur de 0,94 , elles ont une panse plus ou moins ventrue et une contenance qui approche de 30 litres.

Ces amphores portent toutes une marque, la plupart dans un cachet aux angles arrondis sur l'épaule, près de l'attache ou sur l'attache de l'anse (fig. 26), ou bien entre les anses, dans un cachet rectangulaire à la jonction de l'épaule 
et de la panse, au nom de familles connues on Campanic (musée Borély, C. 57-60 ${ }^{40}$. Les marques reconnues sont cclles-ci :

$\mathrm{BAL}$ (bus), DEC(cember) ou DEG(ens), SAL(vius), [V $]^{41}$ ou [C]AR(us), $\mathrm{S}[\mathrm{AT}] 0$, avec la ligature AT (fig. 27).

Cette origine est intéressante pour dater une baignoire-sabot de terre cuite (fig. 28), qui a été remontée, au cours d'un exercice de plongée, par lo GERS



Fig. 29. - La Ciotat. Pattes d'une ancre en fer, d'après le moulage du "creux ", fait par G. Bout (Borély C. 35). Photo Y. Rigoir.

(Ct Pcrrimond). De dimension exiguë, la baignoire très évasée, nc mesure que 0,90 de longucur à la base sur 0,45 de largeur en haut et 0,26 au fond : le dossier relevé est haut de 0,64 , dimensions qui sont à peu de chose près celles des plus anciennes baignoires de terre cuite de Cnossos en Crète, de Mycènes, de Tirynthe, de Santorin. I.e type est en effet connu de la Grèce depuis le xve siècle avant notre

(40) Balbus: CIL, X, 1435-37, 1440, 1471-2 (Herculanum), 2669 (Pouzzoles); December: ibid., 2646 (Pouzzoles), 4460, 4561, etc. (Capoue); ou Decens : ibid., 895 (Pompei); Salvius : ibid., 1032 (Pompei), 2375 (Pouzzoles), 6638 etc. ; Varus : ibid., 885-6 (Pompei) 3351,(Pouzzoles), 3387 (Naples), etc. ou Carus: ibid., 1936 (Pouzzoles).

(41) Cette marque se retrouve au Teslaccio à Rome : CIL, XV, 3230 ; et à Autun : ibid., XIII, 10002,523 . 
ère et s'est perpétué à l'époque hellénistique à Délos, à Priène, à Alexandrie, à Colophon sur la côte ionienne, en Sicile, à Agrigente, à Sélinonte, à Syracuse, etc.

Mais cette baignoire a un fond plat, un trou d'évacuation pour l'eau et ellc est dépourvue de la banquette des baignoires grecques et hellénistiques. Leur exiguïté montre qu'elles avaient pour fonction d'être un bain de pied et un " tub " pour douchage, - " baignoire de propreté », qui a précédé la " baignoire de délassement ", répandue à l'époquc romaine ${ }^{42}$.

De l'épave proviennent également des fragments de tuyau de plomb à soudure latérale (diam. ext. 0,05 ), analogues à ceux qui ont été signalés dans les épaves d'Albenga et du Grand Congloué, et la partic inféricure d'une ancre en fer, dont le bas de la verge présente une double protubérance latérale ct dont les pattes se terminent par un crochet aplati, sur le type des ancres de Pompéi et de $\mathrm{Nemi}^{43}$; le creux conservé sous l'oxydation a permis à M. G. Bout d'obtenir un moulage (fig. 29); le sommet de la verge recevait sans doute un jas mobile et son extrémité inférieure possédait un anneau, qui est conservé sur un autre fragment d'ancre en fer venant de l'épave de Planier A (Borély C. 35 et 41).

4. Bouchons d'amphores. - Très rares sont les bouchons de chaux des amphores qui n'ont pas été corrodés par la mer. Le dévasage des épaves permettra d'en retrouver intacts, comme ceux de $L$. Titi $G$. $f$., incomplets mais restés en place au-dessus du bouchon de liège ou ceux de $M$. C. Lassius, dans l'épave d'Anthéor $\mathrm{A}^{44}$.

Cependant les alluvions du petit Rhône au Grau d'Orgon avaient protégé quelques-uns de ces opercules, au nom de L. Pomponius ${ }^{45}$. D'un gisement d'amphores brisées, mais dont certaines avaient conservé leur bouchon, indice de l'existence d'une épave, proviennent trois bouchons, corrodés, mais protégés par une gangue calcaire de concrétions marines, à la passe de la Jaumegarde, au Langoustier, à la pointe 0 . de l'île de Porquerolles. Aucun débris du col des amphores n'a malhcureusement été conservé (italiques).

Ils présentent la particularité d'être tous les trois estampés avec des cachets en demi-lune ${ }^{46}$, détail technique qui se voit nettement sur les cachets de Lassius, de Pomponius et de Titius; mais peut-être par suite de l'inadaptation de la matrice au diamètre du col, trop étroit, les deux matrices chevauchent l'une sur

(42) Cf. W. Deonna, Mobilier délien, dans Explor. arch. de Délos, 1938, p. 87 et R. Grouvès, Une salle de bain hellénislique à Delphes, BCII, 1952, II, p. 553.

(43) G. UGELl, Le navi di Nemi, p. 238, fig. 270 et 271.

(44) Gallia, VI-1948, p. 213, fig. 8 et. XII-1954, p. 46, fig. 8.

(45) Ibid., VIII-1950, p. 129, fig. 17.

(46) C'est la forme des plus anciens timbres de tuiles et de briques remontant au Haut Empire : G. Lugl, La tecnica edilizia romana (Roma e Lazio), 1957, I, p. 559. 
l'autre, supprimant des lettres ; la restitution peut être faite pour l'un d'entre eux par un second exemplairc. La matière, une chaux d'aspect, blanchâtre, révèle une composition identique, sans trace de quartz ${ }^{47}$ :

1. bouchon de chaux, blanchâtre $(0,095 \text { diam. ; } 0,003 \text { ép. })^{48}$

CA. ISIA. (fig. 30)

2. $i d$. $(0,10$ diam. ; 0,03 ct 0,05 ép. $)$, en double exemplaire ${ }^{49}$

N. Q. SATRI (fig. 31)

Comme pour les précédentes estampilles, il faut noter que celles-ci appartiennent également à la Campanie. Le nom d'Isias se relrouve à Pouzzoles $^{50}$ et celui de Satrius à Herculanum et Capoue ${ }^{51}$.


Fig. 30-31. - Porquerolles. Bouchons d'amphore en chaux d'Isias et Satrius. 1 : 3. Relevé Ch. Lagrand.

C'cst à la Campanie également qu'appartenaient un certain nombre d'estampilles des amphores de l'épave du Grand Congloué et des épaves de La Ciotat et du Dramont. On voit donc l'inlérêt qu'aurait la découverte d'un bouchon marqué appartenant à une amphore estampilléc : le cas ne s'est encore présenté quc pour l'amphore de Sestius, dont le bouchon était au nom de L. Titius. Il ne s'en suit pas que si la marque de l'opercule est celle du productcur, l'estampille de l'amphore soit celle du potier; le "trésor de marques amphoriques » du Dramont nous montre combien est complexe l'interprétation de ces marques qui ne saurait être généralisée. Un cachet sur opcrcule, en terre cuite, au nom de Q. At. Gemellus, en Arles ${ }^{52}$ se retrouve sur des amphores vinaires

(47) L'analyse de M. G. Périnft, ingénicur au Laboratoire de Rayons X du C. R. S. I. M., à Marseille montre qu'ils contiennent $5 \%$ de chlorure de sodium, mais pas de quariz à la différence des bouchons du Grand Congloué. Isias et Satrius : chaux $+5 \frac{1}{2} \% \mathrm{Na}$ Cl. Titius : chaux $+5 \% \mathrm{Na} \mathrm{Cl}+5 \%$ Quartz.

(48) Don du Dr Portaneri, de Toulon (Borély C. 61).

(49) Don du Dr Portaneri et de M. R. Laurent, délégué de la Fédération française d'Études et Sports sous-marins, à Lyon (C. 39 et 62).

(50) CIL, X, 2180, 2451, 2910, 2920, 8194.

(51) Ibid., 1403 (Herculanum), 4329 (Capoue), 5521 (Aquinum).

(52) CIL, XII, 5683, 33 ; cf. 225. 
de Vienne, de Bâle, de $\mathrm{Nyon}^{53}$, - l'estampillage ne pouvant concerner dans les deux cas que le producteur du cru ou le négociant, comme le laisse supposer la contremarque des amphores du Dramont.

Parfois cette marque est anépigraphe : elle est constituée par plusieurs impressions circulaires ou carrées, estampées avant séchage de l'opercule.

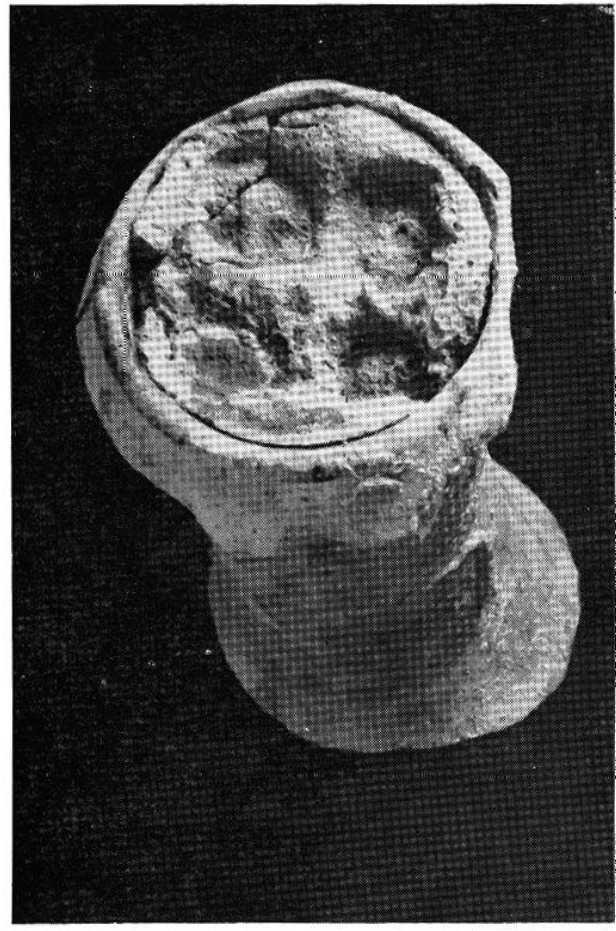

Fig. 32. - Grand Congloué. Bouchon d'amphore gréco-italique à impressions carrées. Pholo Michel Benoit.

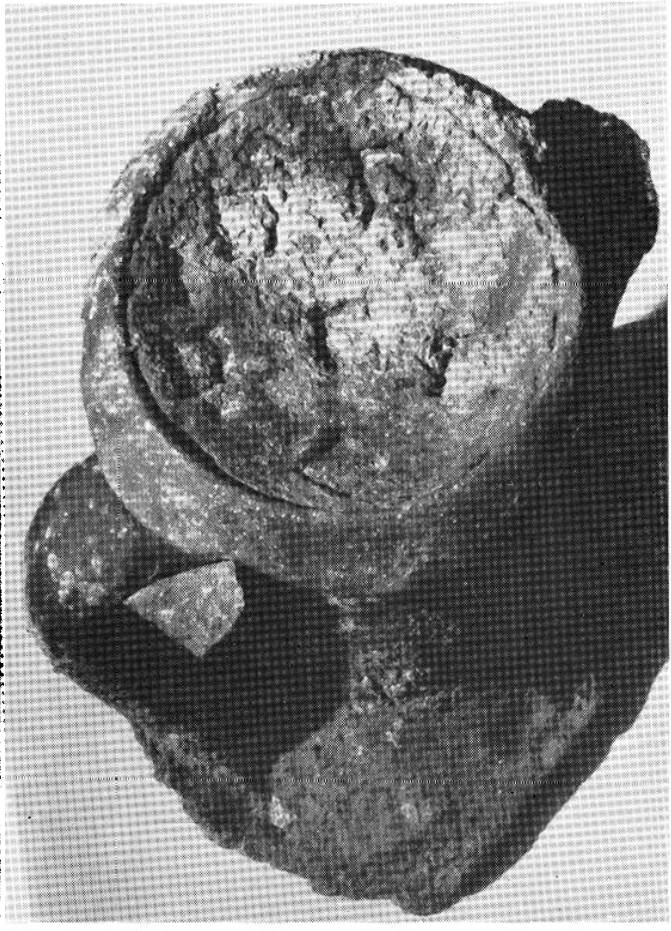

Fig. 33. - Saint-Tropez. Bouchon d'amphore gréco-italique à impressions carrécs. Don $\mathrm{J}$. Courtin (Borély 6790). Photo Y. Rigoir.

Une marque de ce type avait été signalée au Grau d'Orgon ${ }^{54}$, dans un col d'amphore italique ; une autre (impressions carrécs) avait été trouvée à Anthéor, en association avec les bouchons timbrés de Lassius (G. Barnier); cet estampillage est également à noter dans les amphores gréco-italiques, à petite lèvre inclinée $e^{55}$ : au Grand Congloué (fig. 32) (4 impressions rondes Borély LI. I. 351)

(53) Ibid., 5683, 4 et 119 ; XIII, 10002, 243 ; E. PEI.ICHET, Amphores romaines trouvées à Nyons, dans Revue Suisse d'Art el d'Archéologie, VIII, 1946, p. 197, n 50.

(54) Gallia, XIV-1956, p. 224, fig. 12.

(55) Type du II siècle: ibid., XII-1954, p. 37, fig. 2, III ; et XIV-1956, p. 25, fig. 2, 21-22 ; cf. sur ce type Typologie et épigraphie amphoriques, l. c. 
et à Saint-Tropez (fig. 33) sur le haut fonds de la Moutte (6 impressions carrées), riche en fragments dispersés ${ }^{56}$.

5. Épaves du Cap Roux. - Sur la côte de l'Estérel, entre Anthéor et le Trayas, à la pointe du Cap Roux, plusicurs amoncellements d'amphores signalent

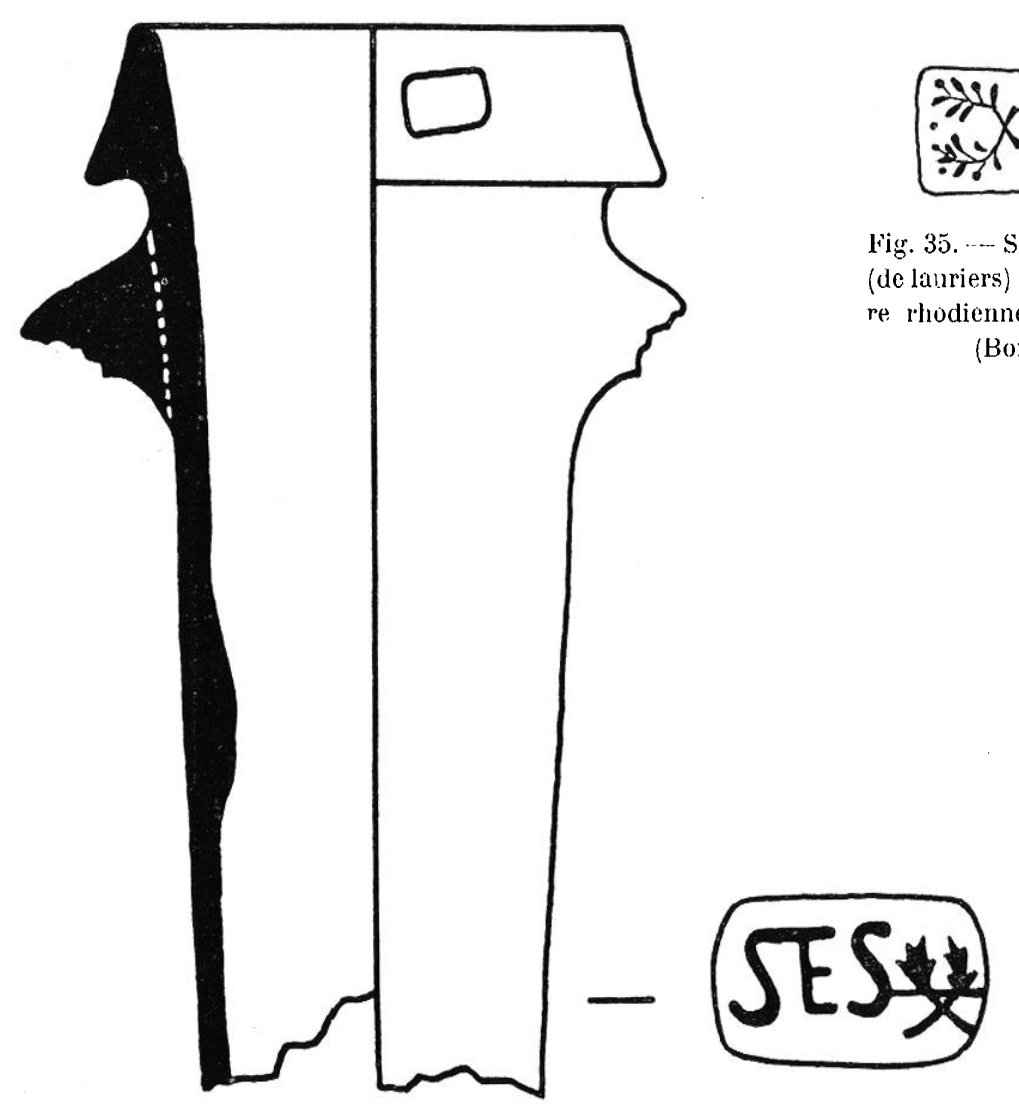

Fig. 34. - Cap Roux. - Amphore italique au timbre de Sestius, suivi de la couronne. 1 : 3. Relevé Ch. Lagrand.

des épaves. A environ 300 mètres de la côte par 18 mètres de fonds, M. J.-J. Egalon a reconnu un champ d'amphores du type Dressel 1. Un col prélevé à titre de témoin porte sur la lèvre un nouveau cachet $(0,029 \times 0,017)$ au nom de Scstius, suivi d'un signe nouveau, une couronne (fig. 34) : la barre supérieure de l'E est liée à la boucle supérieure de l'S, comme dans les marques à l'ancre de Besançon et de Bâle, - défaut d'estampage ou ligature de l'É et du T, ce qui ferait entrer cette marque dans le groupe $\mathrm{SE}(\mathrm{T}) \mathrm{S}$ noté à Alesia ${ }^{57}$.

(56) Trouvée par M. J. Courtin (Borély 6790); cf. sur ce grisemenl, Gallia, XIV-1956, p. 232.

(57) E. Thevenot, La marque d'amphore Sesii, dans Rev. Arch. Est, V, 1954, p. 237, fig. 105, 6. 
Le col tronconique, à large embouchure $(0,14$ diam. $)$, a une lèvre oblique haute de 0,047/0,050; l'attache supérieure de l'anse est nettement détachée des lèvres comme dans le type évolué de l'amphore italique.

Le nouveau signe se réfère comme les autres "symboles" qui suivent ce nom (trident, ancre, caducée, étoile à 5 ou 8 rais, palme, double hache, hameçon ? $)^{58}$ aux signes employés dans les marques grecques des amphores des îles : on trouve la couronne sur les amphores rhodiennes (fig. 35) ${ }^{59}$.
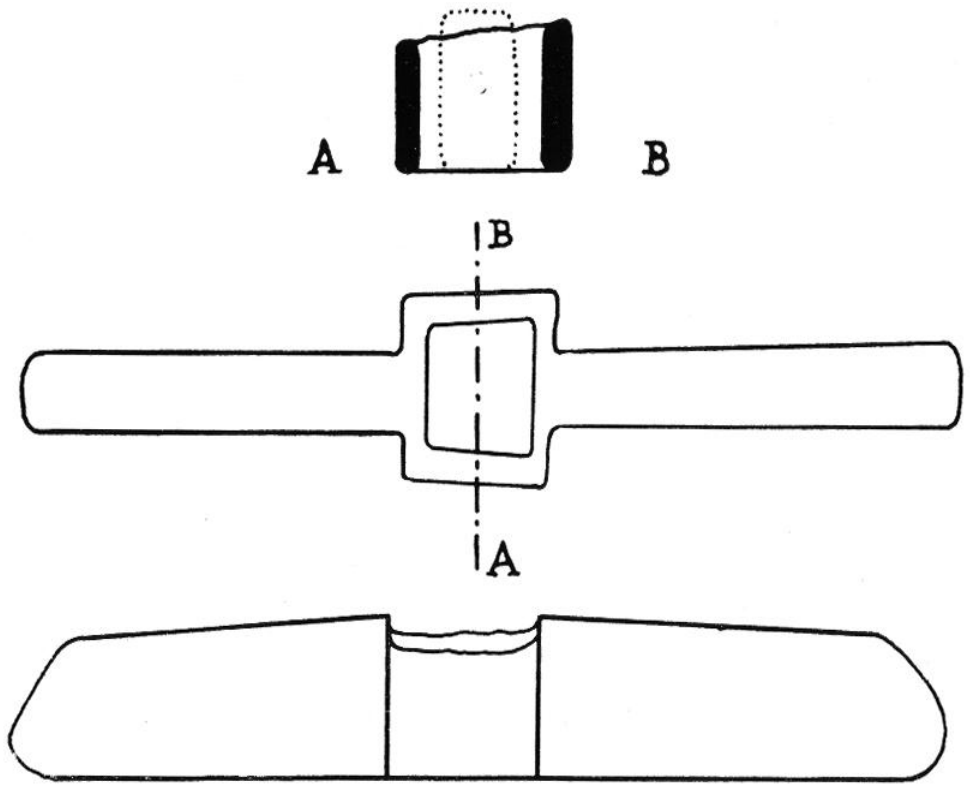

Fig. 36. - Antibes. La Garoupe. Jas d'ancre en plomb présumée de l'épave étrusque. $1: 5$. Fouille du $\mathrm{D}^{\mathrm{r}}$ Pruvot. Rel. Ch. Lagrand.

6. Anlibes. -- La côte du plateau de la Garoupe, découpée par une séric de promontoires, au S.-E. d'Antibes, est un "cimetière marin ». Plusieurs épaves ont pu y être reconnues :

1. Au S., à la Louve, l'épave étrusque découverte par le $D^{r}$ Pruvot ${ }^{60}$ et fouillée avec le concours de M. V. Romanovsky, a donné près de 150 amphores étrusques brisées, de deux dimensions (la plupart du petit modèle) et des fragments de canthares et

(58) Cf. F. Benort, Typologie et épigraphie amphoriques, l. c.

(59) A Carthage: CIL, VIII, 22639, 2 et 51 ; à Istria : V. Caxarache, Imporlul Amforela slampilate la Istria (Biblioteca istorica, I), Bucarest, 1957, no 765 ; à Marseille, fouilles S. Clastrier : ФI $A$ AINOY YAKINOIOY (?) (Musée Borély 9533, 7).

(60) Gallia, XIV-1956, p. 32, no 14 et fig. 6; F. Benort, Relations de Marseille grecque avec le monde occidental, dans Rev. Etudes ligures, 22,1956, p. 20 ; les céramiques dessinées par le $\mathrm{D}^{\mathrm{r}}$ Pruvot et Ch. Lagrand seront publiées par l'inventeur. 
d'œnochoés en bucchero nero, à vernis mat, un fragment de coupe à lèvre courte inclinée, en pâte jaune-clair, bien épuréc, à décor incisé et vernis noir intérieur, de type italocorinthien, une lampe " en bicorne » à deux becs du type dit punique répandu en Méditerranée, en particulier dans les pays d'influence phénicienne (Carthage, Chypre, Malte,

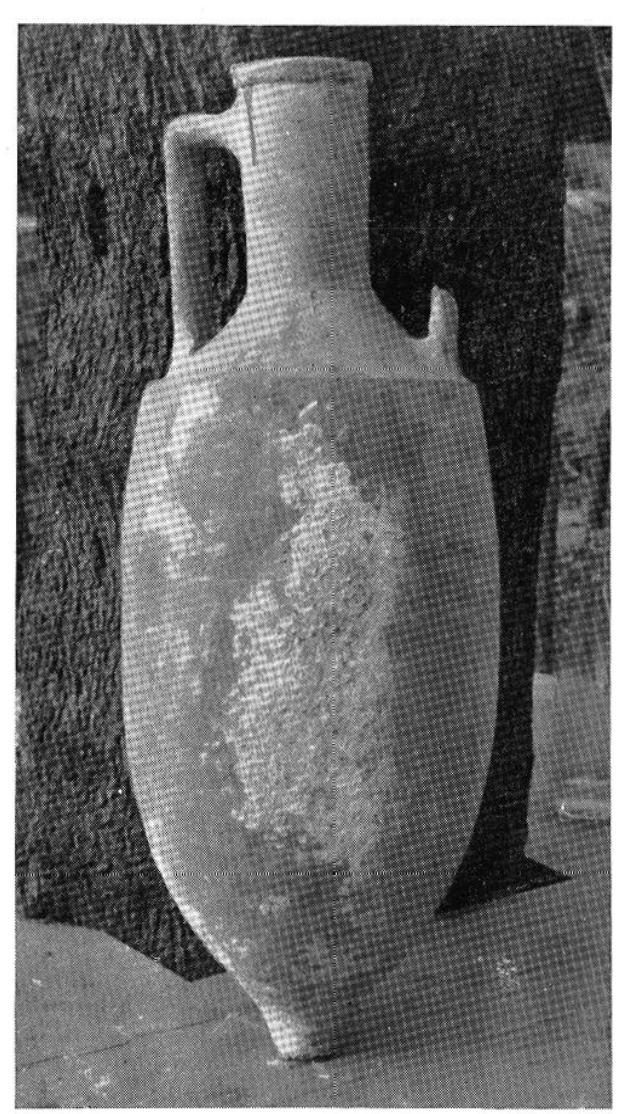

Fig. 37. - I.a Garoupe. Amphore italique à col en bourrelet et anses bifides. Photo $V$. Romanousky.

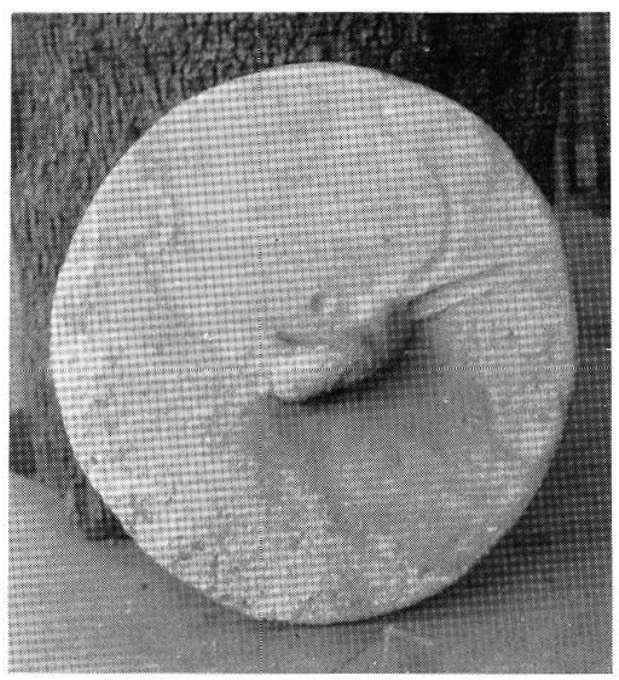

Fig. 38. -... I.a Garoupe. Couvercle de dolium.

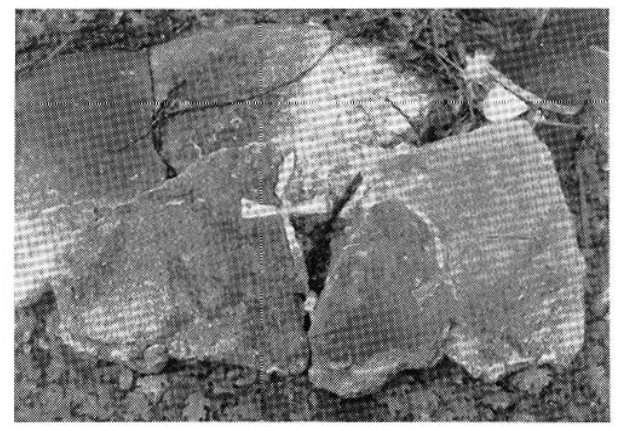

Fig. 39. - La Garoupe. - Fragment de dolium à armature de plomb. Photo V. Romanousky.

Sardaigne) ${ }^{61}$ etc., et un jas d'ancre en plomb, sans armature de bois (fig. 36) de petite dimension, à boîte carrée sans tenon (long. $0 \mathrm{~m}$. 50), qui confirme l'usage de ce type d'ancre au début du vi ${ }^{\mathrm{e}}$ siècle av. J.-G.62 ;

2. plus au N., au Cap Gros, M. Lehoux a reconnu deux épaves, l'une dispersée sur

(61) H. MeNzel, Antike Lampen im Röm. Germ. Zentralmuseum zu Mainz, p. 9, fig. 1; cf. I.. I.f:Rat, Musée de Besangon - Lampes antiques (Annales litt. Université de Besançon, I, 1), 1954, pl. I, l et 2 .

(62) Cf. l'ancre phénicienne de Diodore de Sicile (V, 35, 4), selon l'interprétation de J. Marcadé, appliquée à tort aux pattes de l'ancre $(B C H, 77,1953$, p. 146). 
le tombant de la falaise, par 40 mètres de fond, d'amphores marscillaises à pâte micacée, à rebord droit soutenu par un quart de rond, du vi $\mathrm{e}^{\mathrm{e}} \mathrm{IV} \mathrm{e}^{\mathrm{e}} \mathrm{s}$. av. J.-C. ${ }^{63}$;

3. au promontoire de 'lïrapei, à peu de profondeur (8-12 mètres), à 20 mètres de la falaise, un gisement dispersé, qui présente un grand intérêt par l'association d'amphores et de dolia, pour la date (époque d'Auguste) et la provenance italique de ces derniers :

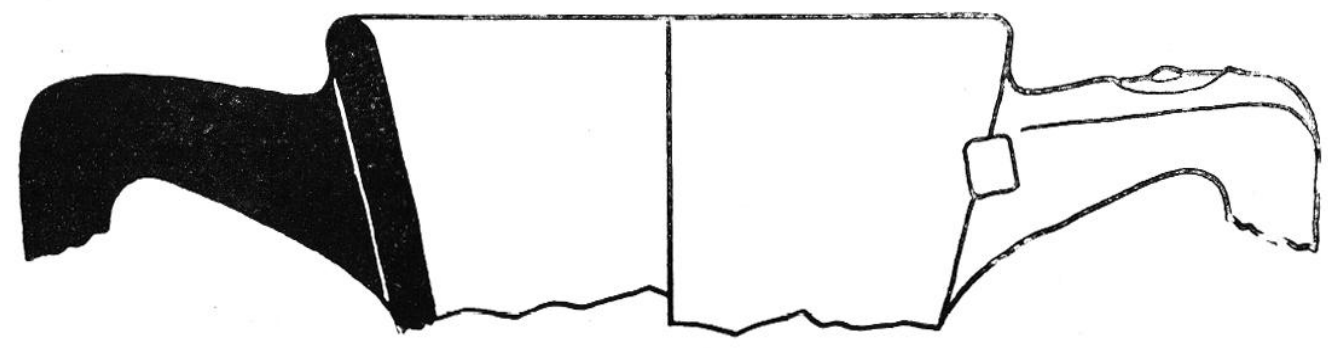

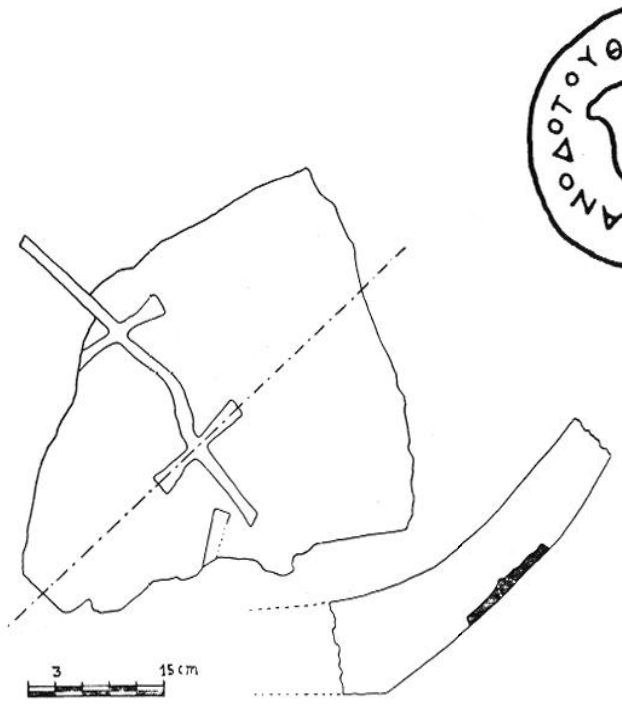

Fig. 10. - Ja Garoupe. - Fond de dolium avec son armature de plomb. Coupe par Ch. Lagrand.
Fig. 41. - Antibes. - Anse StRoch. Eslampille rhodienned'Alhanodolos. Releve Ch. Lagrand.

amphores de tradition grecque à col cn bourrclet ct anses bifides (Dressel 3) (fig. 37), du type de l'épave de Planier $\Lambda$ à Marseille ${ }^{64}$. Ce modèle, de $0 \mathrm{~m}$. 90 environ de hauteur avec le pied, apparaîl donc contemporain des amphores d'Augst, et d'Oberaden, datant de l'époque de la conquête, selon la classification de Loeschke ${ }^{65}$, el suit la tradition plus ancienne, représentée par des amphores à anses pleines en boudin, datées par les

(63) Gallia, l. c., p. 33, no 15 et fig. 1, no 4.

(61) Ibid., p. 26, no 1 et fig. $1, n^{\circ} 7$.

(65) Cf. E. Gose, Gefässlypen der räm. Keramik im Rheinland, Bonn, 1950, pl. 59, no 434 (Oberaden).

Fig. 12. - Anse St-Roch. -- Estampilles rhodiennes avec le soleil radić. Relevé Ch. Lagrand. 
marques rhodiennes de l'anse de la dernière période des timbres amphoriques (fin II $^{\mathrm{e}_{-}}$ début $\mathrm{I}^{\text {er }}$ s. av. J.-C. $)^{66}$;

grands dolia en terre cuite sans bourrelet de renfort à la base (haut. env. $1 \mathrm{~m} .65$; ép. 0 m. 07), présentant une armature de plomb constituée par des agrafes encastrées dans la paroi, en forme de patte munie de tenons latéraux en double queue d'aronde (fig. 39 et 40) ; le même dispositif avait été reconnu dans les dolia en forme de pithos (à bourrelet de renfort à la base) des docks romains de Marseille. Il ne s'agit donc point de réparation en cours d'usage, mais d'un renforcement du récipient, à la fabrique.

Avec ce chargement, ont été trouvés un couvercle de dolium en terre cuite (fig. 38), avec anse de préhension (diam. $0 \mathrm{~m}$. 45 ; ép. $0 \mathrm{~m} .03$ ), un pelvis (diam. $0 \mathrm{~m} .40$ ), un récipient en plomb de forme hémisphérique, sans doute une urne funéraire (diam. $0 \mathrm{~m} .46$; haut. env. 0 m. 25);

4. une autre épave au S.-E. de l'îlot de la Grenille, contenant de la vaisselle campanienne, a été signalée "aux Berges", au large du Cap Gros, par 65 mètres de fonds.

Anse Saint-Roch. - L'anse Saint-Roch, en cours d'envasement, est également très riche. Nous avons signalé une épave du Bas-Empire, englobée dans une motte, vers la passe de l'ancien port, aujourd'hui atterri entre le Fort Carré et le littoral O. de l'anse ${ }^{67}$. Une seconde épave a été signalée au N.-O. de celle-ci.

$\Lambda u$ fond de l'anse, au N. de la "table Saint-Roch ", dalles appartenant à l'ancien quai, aujourd'hui entourée d'eau par suite de l'érosion de cette partie de l'anse, la prospection de M. J. Marguier montre l'existence d'un gisement envasé à peu de profondeur (1 mètre à $1 \mathrm{~m} .50), \cdots$ dépotoir ou épave. Il a y recueilli un grand nombre de fragments d'amphores italiques, appartenant principalement à la forme Dressel $1 \Lambda$ et d'amphores rhodiennes, avec marques sur le coude de l'anse. La datation de ces dernières amphores de la fin du III $^{\mathrm{e}}$ s.-début II ${ }^{\mathrm{e}}$ siècle est intéressante à noter, par son synchronisme avec celle des amphores du Grand Congloué à Marseille ${ }^{68}$, des marques trouvées dans les fouilles terrestres de Marseille ${ }^{69}$, d'Olbia, d'Entremont, d'Ensérune, de Narbonne, etc. :

a) 3 marques circulaires à la rose, sur coude d'anse presque horizontal ${ }^{70}$, dont une lisible donnant l'éponyme, suivi du nom du mois :

\section{[E]חI AOANOAOT[OY $\Theta] E \Sigma M O[\Phi]$ OP [IOY[ (fig. 41) $)^{71}$.}

b) 20 marques rectangulaires :

une lisible au nom du fabricant: $\Delta \mathrm{l}[\Sigma \mathrm{K}] \mathrm{OY}^{72}$, à rapprocher d'une marque sans prove-

(66) J. Mrrtens, Les fouilles d'Alba Fucens, Bruxelles, 1955, p. 152 et fig. 35.

(67) Gallia, XIV-1956, p. 34, no 16 et Rev. Et. ligures, 18, 1952, p. 256. Voir le plan d'Antibes dans les Informations, circonsc:iption d'Aix (Sud), plus loin.

(68) Gallia, XII-1954, p. 38, n. 5 ; ct Synchronisme des amphores rhodiennes el de la céramique hellénistique de l'épave du Grand Congloué, dans Rhodania, 29-30, 1954-1955, p. 9-10.

(69) Coll. Clastrier : fouilles de l'ancien couvent des Grandes Maries, rue des Phocéens (Mém. Instilut historique de Provence, X, 1933, p. 27 ; XII, 1935, p. 10, n. 1 : musée Borély 9533 et sans provenance 5580).

(70) Voir sur ces profils V. Grлc., Stamped Amphora Handles, dans Hesperia, 3, 1934, pl. II.

(71) V. Grace, T'imbres amphoriques trouvés à Délos, l. c., p. 528 (220-180 av. J.-C.).

(72) A. Maiuri, Una fabbrica di anfore rodie (Scuola arch. di $\Lambda$ tene, IV-V) 1924, p. 263. Cf. V. GraGe, The Eponyms named on Rhodian Amphora Slamps, dans Hesperia, 22, 1953, pl. 42. 
nance du musée de Besançon ${ }^{73}$; une seconde incomplète avec le soleil radié et une marque éponyme incomplète sur deux lignes [APX]OKPATE[Y $[$ ] | En|.... (fig. 42a) (haut. : 15 millimètres) ${ }^{74}$, et les symboles usuels dans les amphores rhodiennes : corne d'abondance ${ }^{75}$, soleil radic ${ }^{76}$.

\%. Champ d'amphores et épave de Fos. - L'exploration de l'anse SaintGervais à Fos par la "Société des Amis du Vicil Istres" dirigée par le Dr Bcaucaire, à laquelle s'était joint le groupe de P. Delange, R. Diot, M. Michaud et Cl. Thomas, a été particulièrement fructueuse (fig. 43).

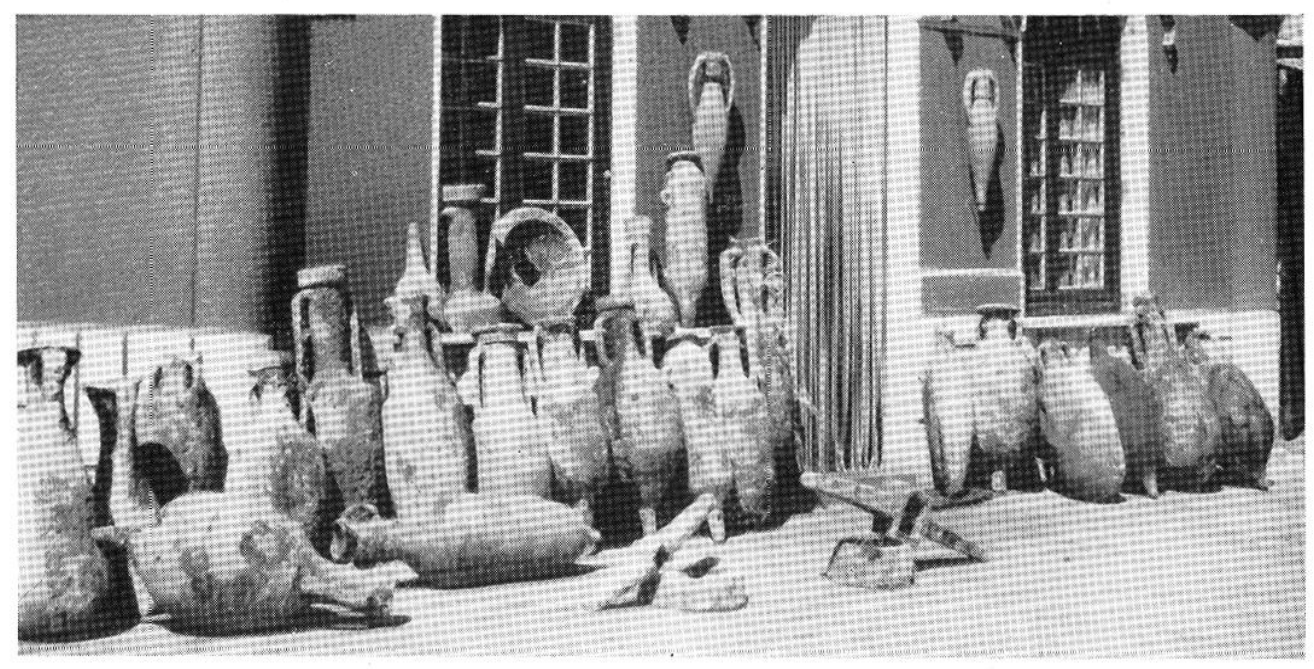

Fig. 43. - Fos. - La récolte d'amphores et jas d'ancres d'une saison. (Les amphores scellées au mur sont en ciment). -- Musée du Vieil-Istres. Photo R. Diol.

En raison du peu de profondeur, les différents types d'amphores remontées ne peuvent permettre de localiser une épave à proximité du littoral ${ }^{77}$. $\Lambda$ signaler des amphores du type d'Augst, à col très large monté sur une panse piriforme à pied massif élevé et lèvre évasée (fig. 44) : ce type très rare en Narbonnaise (des exemplaires à Arles) et

(73) Je dois à l'obligeance de M. Lerat la connaissance de ces deux anses, sans provenance, appartenant au vieux fonds du muscé ; l'autre marque est au nom de l'éponyme [ANAミ ?] AN $\triangle P O Y$, suivi du nom du mois ATPIANIOY, qui d'après V. Grace appartiendrait à la deuxième moitié du $I^{\mathrm{e}}$ siècle.

(74) Je remercie M. I. Benachi de son identification.

(75) C. V. Grace, Timbres amphoriques, l. c., p. 536, no 18 et pl. 21 ; V. Caxarache, Imporlul Amforela slampilale la Islria, no 566.

(76) A Rhodes : Canaracire, o. c., no 595 ; à Thasos, ibid., no 116 ; à Rhodes sur anses bifides analogues à celles du Grand Congloué (comm. de M. L. Benachi); plusieurs marques de la Coll. Clastrier à Marseille portent ce symbole suivi d'un éponyme : Eח। EENOФANEY $\Sigma$, EחI $\Sigma \Omega \Sigma T P A T O Y$ (Musée Borély 9533, 4 et 8) de 220-180 av. J.-C. (V. (Grace, Timbres amphoriques, p. 529-530) et soleil radié avec caractères grecs entre les rayons (Borély 5580), analogue à une marque de Rhodes publiée par Mailiri (l. c., p. 268, fig. 9).

(77) Cf. Gallia, VIII-1950, p. 123 ; XI-1953, p. 111 ; XII-1954, p. 432. 
nouveau sur la côte est au contraire caractéristique des gisements des camps du Rhin (Auguste à Claude) ${ }^{78}$; des inscriptions peintes sur des amphores de ce lype à Augst indiquaient qu'elles avaient contenu du garum; on en rapprochera les exemplaires de Nyon et d'Hofheim (forme 46 de Pélichet) ${ }^{79}$;

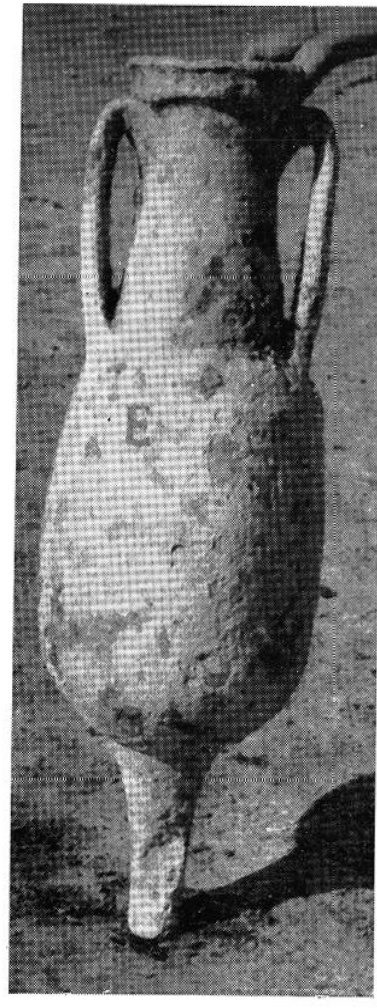

Fig. 44. - Fos. - Amphore du type d'Augst (haut. $0 \mathrm{~m} .84$ ). - Musće du Vieil-Istres. Pholo R. Diot.

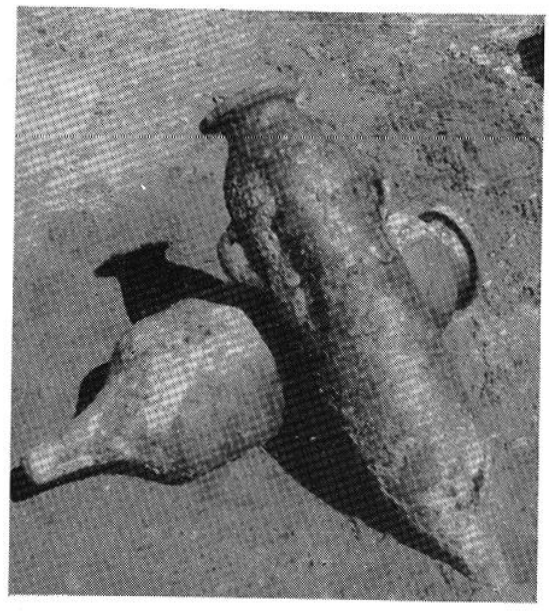

Fig. 45. - Fos. - Amphores du type dit punique. - Muséc du Vieil Istres. -... Photo R. Diot.

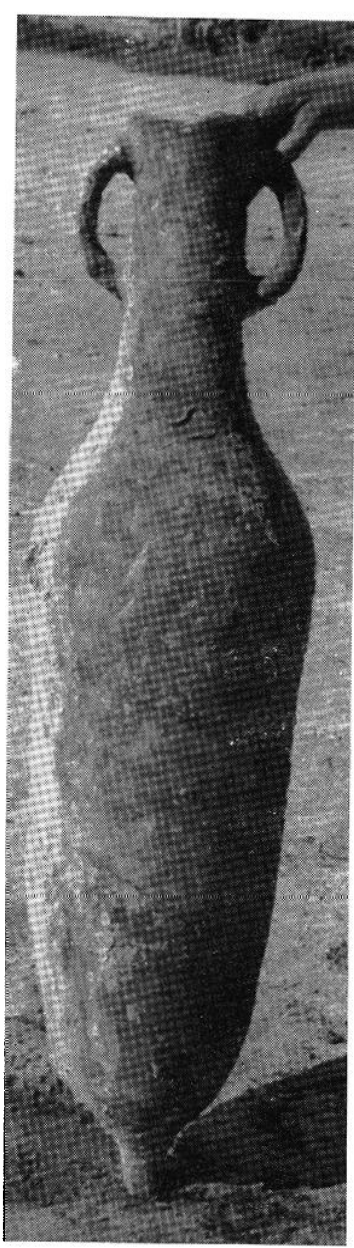

Fig. 46. - Fos. - Amphorc à deux petites anses au haut du col. - Musće du Vieil-Istres. Photo R. Diot.

des amphores ilaliques de haute taille (haut. : $1 \mathrm{~m} .20$ ), du type Dressel $1 \mathrm{~B}$, caractéristique du milieu du $\mathrm{I}^{\mathrm{er}} \mathrm{s}$. ;

des amphores dites de type punique à panse cylindrique (fig. 45) munies de deux petites anses au haut de la panse, à col court et collerette droite ou biseautée, que nous

(78) A. Grenier. Man. Arch. gallo-romaine, II, p. 615, fig. 210 et p. 635. fig. 435 .

(79) E. PéLIGHET, Amphores romaines lrouvées à Nyon, l. c., p. 192, fig. 3 ; E. Gose, l. c., pl. 60, 
avons comparées à l'exemplaire du Dramont, de date un peu moins ancienne ${ }^{87}$; et un exemplaire, dont les petites anses rondes sont reportées au haut du col (fig. 46) dépourvu de sa collerette;

deux jas de plomb, l'un de $0 \mathrm{~m}$. 60 de long (poids 21 kilos) avec bois sans tenon de fixation; l'autre de $1 \mathrm{~m}$. 07 de long (poids 84 kilos), portant une marque incisée sur l'un des còtés (fig. 47), en capitales très régulières alignées entre deux traits gravés, les $\mathrm{G}$ étant faits au compas :

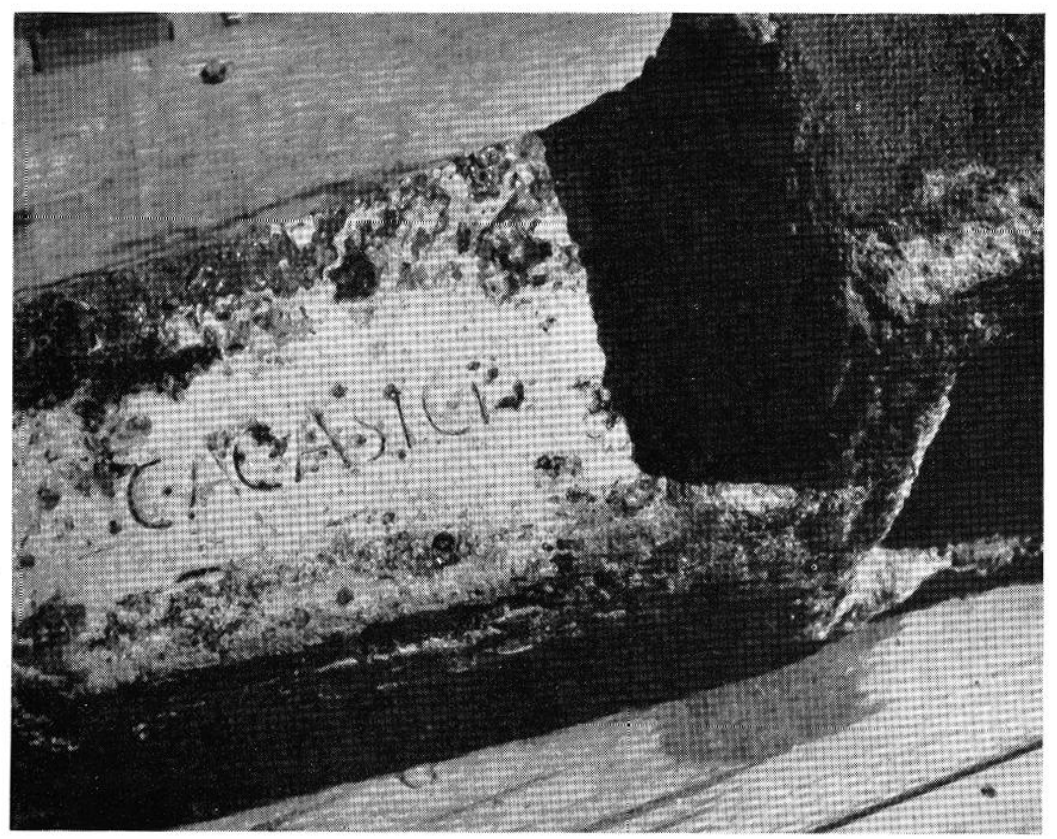

Fig. 47. - Fos. - Jas d'ancre avec la marque d'Asicius. - Musée du Vieil-Istres. - Pholo R. Diol.

\section{G. A G. A S I C I}

C. Ac(ilius) Asici[us? $]^{81}$

haut. des lettres : 20 millimètres ; dernière lettre incomplète du haut;

un saumon de plomb de 66 kilog. en forme de parallélépipède tronqué $(0 \mathrm{~m} .535$ longueur à la basc et $0 \mathrm{~m} .185$ au sommet, et $0 \mathrm{~m}$. 140 et $0 \mathrm{~m} .070$ de largeur sur $0 \mathrm{~m} .12$ de hauteur), dimensions et poids qui l'apparentent aux lingots d'origine britannique, plus volumineux que ceux d'Espagne et de Sardaigne qui pèsent une centaine de livres ${ }^{82}$.

(80) Type des amphores de l'agora S S 14069 et P 6762 : V. Grace, The Canaanite Jar, l. c.; supra, $\mathrm{n}^{\circ} 2$.

(81) C. en Italie la gens Acilia: CIL, X, 6130, 8053, ${ }^{221}, 8056,{ }^{402}$, etc. ; et le cognomen Asicius (Asic. of.) sur un vase de Tarragone : $C I L$, II, 4970, 50.

(82) Cf. A. BESNIER, Le commerce du plomb à l'époque romaine, dans Rev. arch., 1920, II, p. 222, 232. 
Sur la tranche supérieure une marque estampée en relief dans un cadre :

\section{S O G I O R UM P L U M B (ariorum) G É R (mani) $)^{83}$}

hauleur des lettres 27 millimètres; $\mathrm{S}$ inversé ; ligature PL. Sur le petit côté de gauche un chiffre gravé X L V I I, ne correspond pas au poids.

Non loin de ce lingol, a été repérée une épave reconnaissable au groupement des amphores, recouvertes par le sable, par 8 à 10 mètres de fonds (MM. Fournon, Agnel, Illaret). Quelques fragments de bordés avec assemblages mortaisés et clous de fer, des poteries communes (poterie en terre rouge à rebord droit, avec graffite $\mathrm{M} \mathrm{S}$, bols et couvercles corrodés) appartenaient à l'épave qui a été dispersée.

Le type d'amphores Dressel $1 \mathrm{~B}$, de $1 \mathrm{~m} .10$ à $1 \mathrm{~m} .20$ de haut, dont le col avait parfois conservé un restant de bouchon de chaux, est caractérisé par son aspect massif et par le raccord à angle presque droit de l'épaule avec la panse, qui les rapprochent de celles du Dramont; la lèvre verticale, de $0 \mathrm{~m}$. 05 de haut. porte sur trois exemplaires une estampille dans un cachet rectangulaire :

M $\Lambda \mathrm{HE}$ avec ligature des deux dernières lettres ${ }^{84}$, M I ${ }^{85}$ et $S \Lambda M\left(\right.$ (?) $I^{86}$, qui peuvent être rapprochées de marques de potier de Narbonnaise et de Tarraconnaise.

8. Iles d'Hyères. - Les côtes de l'île de Port-Cros sont riches en débris d'amphores, qui indiquent peut-être des épaves dispersées. A signaler sur la "sèche " de la Gabinière, au S. de l'île, par 35 mètres un jas d'ancre en plomb (long. 0 m. 59), remonté par M. Pinard, de l'École de Navigation sous-marine (Musée de la Marine à Toulon); sur le tombant de l'îlot de Bagau, au N.-E. par 15 mètres de fond, plusieurs cols d'amphores, parmi lesquels un col d'amphore rhodienne à anse remontante faisant un coude aigu (fig. 48) et un grand pelvis très profond, avec égouttoir (diam. 0 m. 63 ; haut. : 0 m. 20). (Cdt Perrimond, au Gers.)

9. Rade de Villefranche-sur-Mer. -- La prospection de la rade de Villefranche, qui a déjà donné d'importants résultats ${ }^{87}$, sans permettre loutefois de localiser une épave, a permis à M. Éd. Allemand, du Club de l'Exploration sous-marine de Nice, de remonter deux jas d'ancre en plomb dont l'un de $1 \mathrm{~m}$. 15 de longueur, est orné de quatre osselets apotropaïques, disposés par paire sur chaque bras du jas. Ces nouvelles pièces portent à cinq le nombre de jas retirés de la rade (fig. 49). La marque de celui-ci permet de le rapprocher du jas de Carry-le-Rouet, au musée Borély ${ }^{88}$, l'un des plus grands connus (long. $2 \mathrm{~m} .16$ ), et d'un jas mobile, découvert au large de Syracuse (long. 0 m. 70) ) $^{89}$, tous deux ornés de quatre osselets disposés sur la même tranche du bras du jas.

(83) Cif. sur ce nom D. Rendré-Mročevré, Neue epigraphische Belege für den Namen Germanus im illyrischen Namengut Dalmatiens, dans Germania, 34, 1956, p. 237. 291 '.

(81) Plusieurs variétès : Mahe(tis) : CIL, XII, $5686,{ }^{523}$; à Tarragone : Mahe(s) : ibid., II, 4970,

(85) A Fréjus : CIL, XII, 5686, 524.

(86) Cf. CIL, X, 3537 (Samus).

(87) Gallia, XIV-1956, p. 34, no 17.

(88) W. Froenner, Musée de Marseille, Cal. des Antiquités grecques et romaines, 1897, n 17/1517.

(89) F. Benort, Jas d'ancre el pièces d'outillage des épaves de Provence, dans Rev. Éludes ligures, XXI, 1955, p. 123, fig. 7 et 8.

(90) Gallia, XIV-1956, p. 239. 
10. Corse. -- La coordination des recherches sous-marines faites dans le Sud de la Corse par M. R. Grosjean ct ses reconnaissances en plongée lui ont permis de localiser des mouillages par les débris d'amphores avoisinant le port et de préciser les gisements appartenant aux épaves du "cimetière marin " des îles Lavezzi, dans le détroit de Bonifacio :
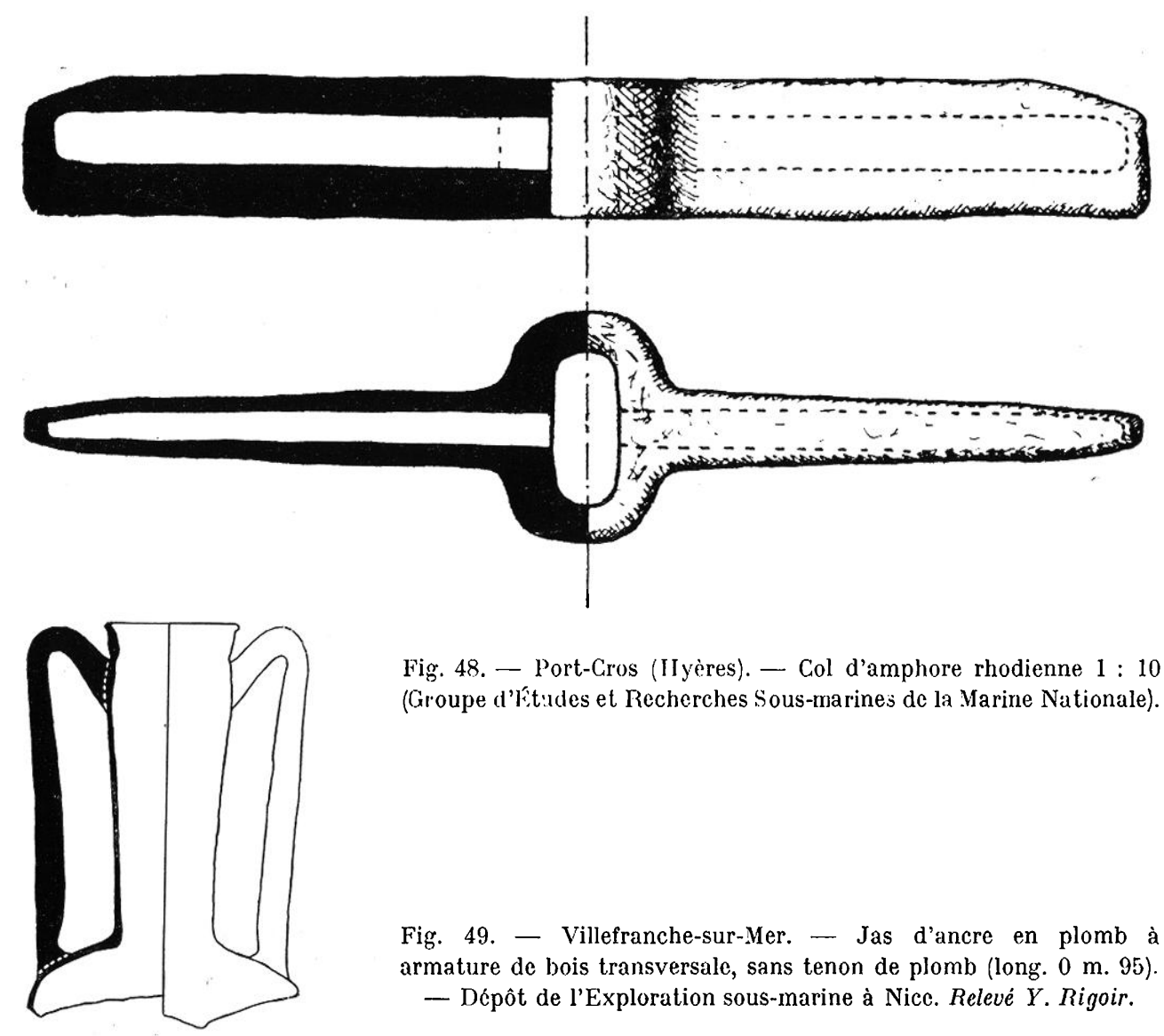

Fig. 48. - Port-Cros (IIyères). - Col d'amphore rhodienne $1: 10$ (Groupe d'lit:udes et Recherches Sous-marines de la Marine Nationale).

Fig. 49. - Villefranche-sur-Mer. - Jas d'ancre en plomb à armature de bois transversale, sans tenon de plomb (long. $0 \mathrm{~m} .95$ ). - Dépôt de l'Exploration sous-marine à Nicc. Relevé Y. Rigoir.

1. Porto-Pollo (comm. de Serra di Ferro), sur la côte N. du golfe de Valinco, au N. de l'embouchure du Taravo : amphore à huile (Dressel 24); jas d'ancre mobile et pièce d'assemblage en plomb;

2. Tizzano, Tilianus porlus de Ptolémée : amphores Dressel 14 et cols à lèvres évasées ịtype $\mathrm{F}$ de Pélichet) ;

3. Figari (Figaria) ${ }^{90}$ : le port antique se trouverait à l'abri d'une ligne de rochers faisant l'office d'une digue, à une profondeur d'environ 5 mètres ;

4. Ile Cavallo: au pied du rocher de San Baïnzo, plusieurs amphores gréco-italiques à col en bourrelet et anses bifides (Dressel 2-3) ${ }^{91}$;

(91) Ibid., p. 24, fig. 1, nos 6-10. 
5. Cap Sperone, à la balise du Prêtre : plusieurs épaves avec amphores de types différents : col rhodien (?), gréco-romaine ${ }^{92}$, dont une avec marque $M A H E$ (s) sur le haut de la panse ${ }^{93}$, italique (Dressel 1), sphérique (Dressel 20), cylindrique ${ }^{94}$, byzantine ; 2 flans de cuivre, en forme de disque, de 50 kilos environ;

6. Iles Lavezzi: amphores italiques à panse ventrue (Dressel 8).

Fernand Benoit.

(92) Gallia, XIV-1956, p. 25, fig. 2, nos 21, 22.

(93) Voir supra, n. 84.

(94) Gallia, ibid., fig. 2, no 27. 